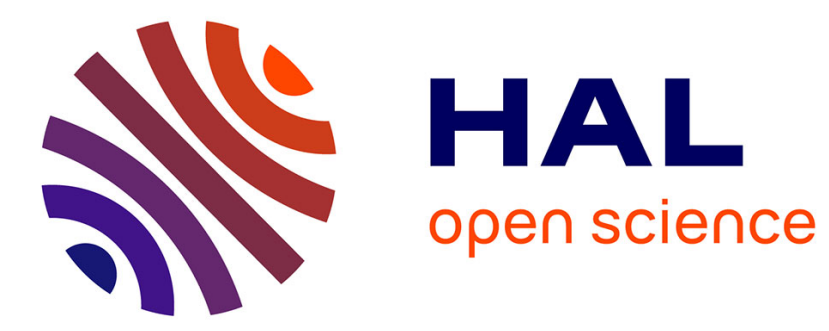

\title{
Dimensionnement d'une machine a commutation electronique monophasee haute vitesse à réluctance et aimants alternés
}

\author{
A. Ben Ahmed, E. Aufauvre, Bernard Multon
}

\section{- To cite this version:}

A. Ben Ahmed, E. Aufauvre, Bernard Multon. Dimensionnement d'une machine a commutation electronique monophasee haute vitesse à réluctance et aimants alternés. Journal de Physique III, 1997, 7 (10), pp.2031-2058. 10.1051/jp3:1997240 • jpa-00249699

\section{HAL Id: jpa-00249699 https://hal.science/jpa-00249699}

Submitted on 1 Jan 1997

HAL is a multi-disciplinary open access archive for the deposit and dissemination of scientific research documents, whether they are published or not. The documents may come from teaching and research institutions in France or abroad, or from public or private research centers.
L'archive ouverte pluridisciplinaire HAL, est destinée au dépôt et à la diffusion de documents scientifiques de niveau recherche, publiés ou non, émanant des établissements d'enseignement et de recherche français ou étrangers, des laboratoires publics ou privés. 


\title{
Dimensionnement d'une machine à commutation électronique monophasée haute vitesse à réluctance et aimants alternés
}

\author{
A. Ben Ahmed $\left({ }^{1, *}\right)$, E. Aufauvre $\left({ }^{1}\right)$ et B. Multon $\left({ }^{1,2}\right)$ \\ $\left({ }^{1}\right)$ Laboratoire d'Électricité, Signaux et Robotique $\left(\operatorname{LES}_{1} R\right)\left({ }^{* *}\right)$, École Normale Supérieure de \\ Cachan, 61, avenue du Président Wilson, 94235 Cachan Cedex, France \\ $\left({ }^{2}\right)$ Antenne de Bretagne de l'ENS, campus de Ker Lann, 35170 Bruz, France
}

(Reçu le 18 novembre 1996, révisé le 16 juin 1996, accepté le 18 juin 1997)

PACS.02 $60-x$ - Numerical approximation and analysis

\begin{abstract}
Résumé. - Les auteurs présentent dans cet article le dimensionnement d'une machine monophasée à aimants alternés statoriques à bobinage global et à circurt magnétique massif réalisé à partır de poudre de fer. La méthode utilisée combine un calcul numérıque par éléments finis et un calcul analytique en régime linéaire. Le dimensionnement complet notamment la détermination des couples instantanés prenant en compte l'encombrement global de la machıne, les pertes Joule et fer ainsı que les conditıons de démarrage est effectué. Après avoir défini le modèle de calcul à partir d'un schéma magnétique équivalent, une étude paramétrique est réalisée Celle-ci a pour but d'étudier l'influence de certains paramètres fondamentaux tels que la largeur angulaire des dents rotoriques sur les performances de la machine et particulièrement sur les conditions de démarrage en mode monophasé. Les résultats théoriques sont ensuite confrontés aux relevés expérimentaux effectués sur plusieurs prototypes de machines à aimants ferrites.
\end{abstract}

\begin{abstract}
In this paper, the authors present a design methodology of single phase reluctance permanent magnet machmes with centralised coil The massif magnetic circuit is realised using iron powder. The used method is a combination of a finte element numerical calculation and an analytic one in a linear regime The complete design is then done, especially the instantaneous torque determination considering the machine's global dimensions, iron and copper losses, and the starting conditions. A parametrical study is realised after defining the computation model from an equivalent magnetic circuit. This study aims to analyse some fundamental parameter's influence on the machine's performances and particularly on the starting conditions in single phase mode. These parameters are such as rotor tooth angular width. The theoretical results are then compared to the experimental ones obtanned on several ferrite magnet machine prototypes.
\end{abstract}

\section{Introduction}

Depus quelques années, le besoin en petit moteurs rapides ( $>10000 \mathrm{tr} / \mathrm{min}$ ), fiables et économiques s'est énormément accru dans de nombreux secteurs de l'équipement grand public (électroménager, bureautique, ...) mais également industriel (machine-outil, automobile, médical, ...) $[12]$. Par ailleurs, les progrès technologiques intervenus dans des domaines tels que celui

(*) Auteur auquel doit être adressée la correspondance (e-mail . benahmed@lesir ens-cachan.fr)

$\left({ }^{* *}\right)$ URA CNRS D1375

(C) Les Éditions de Physique 1997 
des aimants [2], de l'électronique de puissance (haute fréquence) et de son intégration favorisent aujourd'hui la naissance de nouveaux actionneurs présentant des structures originales [4].

Les topologies monophasées présentent, pour des faibles encombrements, plusieurs avantages, notamment la simplicité de la fabrication, en outre l'électronique d'alimentation et de commande peut être facilement intégrée. Néanmoins, les structures monophasées actuelles utilisent pour le démarrage des artifices extérieurs électriques ou magnétiques tels les condensateurs, les pôles auxiliaires de démarrage [13], les dissymétries magnétiques (entrefer variable [10,11]). La plupart de ces structures exigent donc des composants magnétiques ou électriques supplémentaires engendrant une augmentation du coût, une diminution du rendement et de la fiabilité de fonctionnement. De plus, les dissymétries d'entrefer sont souvent sources de vibrations et de bruit acoustique.

Un autre procédé de démarrage en monophasé peut être cependant utilisé. En effet, une machine à aimants sans variation de l'inductance propre est le siège d'un couple électromagnétique comprenant deux composantes. La première est le couple hybride, fonction du courant d'alimentation, à valeur moyenne non nulle produisant la puissance mécanique, la seconde est le couple de détente à valeur moyenne nulle représentant l'interaction des aimants permanents avec le circuit magnétique. Ce couple possède généralement, une fréquence double de celle du couple hybride. Sur un pas, le couple de détente s'annule quatre fois engendrant quatre positions d'équilibre dont deux seulement sont stables habituellement (voir Fig. 1a). Les positions stables correspondent aux positions de couple hybride nul, le démarrage monophasé ne peut donc s'effectuer. Maintenant, si l'on inverse la forme d'onde du couple de détente, autrement dit si l'on stabilise les positions précédemment instables et si l'on déstabilise les positions précédemment stables (voir Fig. 1b), l'injection d'un courant dans la bobine crée un couple hybride non nul et permet ainsi le démarrage.

Ce procédé [4] présente les avantages suivants :

- une seule bobine;

- aucune dissymétrie magnétique ;

- pas d'artifice électrique extérieur ;

- pas de surdimensionnement des aimants permanents (pas de surintensité nécessaire au démarrage) ;

- fiabilité de fonctionnement accrue.

L'inversion de l'allure du couple de détente est obtenue par modification de la géométrie des aimants, des dents rotoriques et du jeu mécanique. Cette modification entraîne, a pron une désoptimisation du flux inducteur et, ainsi, une diminution des performances de la machine. Nous montrons, dans cette structure particulière, que la perte de performances est très faible et nous présentons dans cet article, les proportions de cette diminution, l'influence des différents paramètres géométriques sur le couple de détente, sur le couple moteur moyen et sur les pertes Joule et magnétiques.

\section{Modèle de dimensionnement}

Une modélisation entièrement analytique permet de faciliter la compréhension des principaux phénomènes présents au sein de la machine, elle ne peut pas en revanche être utilisée de manière fiable pour optimiser notre structure, en particulier dans le calcul du couple de détente 


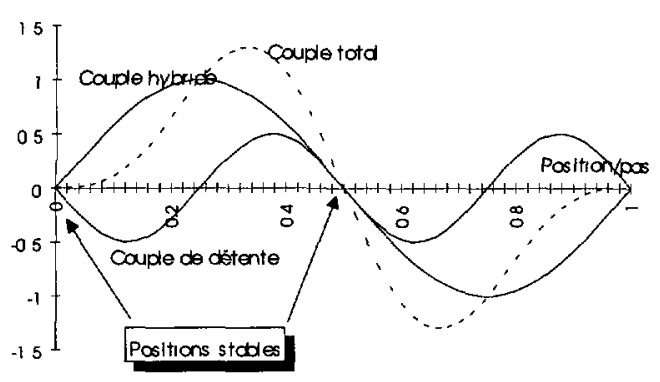

a)

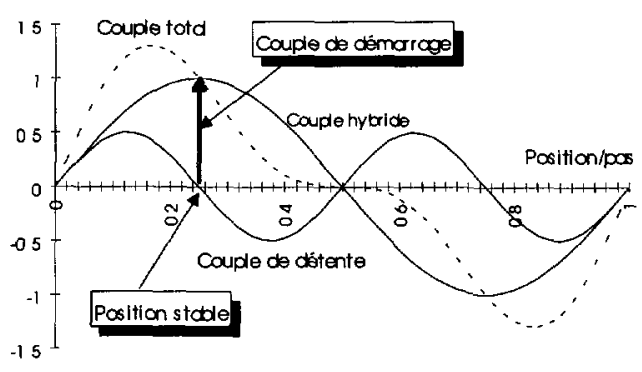

b)

Fig 1. - Exemple de variation des couples électromagnétiques en fonction de la position du rotor pour un courant d'alimentation monodirectionnel. a) démarrage en monophasé impossible, b) démarrage possible.

[Instantaneous electromagnetic torque waveform example. a) impossible starting in a single phase case, b) possible starting case.]

instantané à cause de la très grande sensibilité du couple de détente à la répartition du champ magnétique d'entrefer.

Une méthode entièrement numérique [9] se révèle lourde dans le cas étudié notamment à cause des effets tridimensionnels. D'autre part, lorsqu'il est nécessaire d'optimiser un système complet, les calculs $3 \mathrm{D}$ numériques deviennent très longs et demandent un investissement matériel et temporel important, souvent démesuré par rapport au problème posé.

Il se révèle que le développement de programmes spécifiques, alliant les calculs analytiques et numériques par éléments finis, permet de combiner souplesse, rapidité d'exécution et précision $[6,7,15,17,18]$.

Tout d'abord, nous présentons la structure du schéma magnétique équivalent que nous avons retenue et les méthodes de calcul permettant de déterminer les paramètres qu'il comporte. Nous pourrons alors établir les différentes relations permettant de calculer les principales performances et caractéristiques de la machine.

2.1. STRUCTURE ÉTUdiÉE ET HYPOTHÈSES DE CALCUL . - La machine étudiée est composée $[5,16]$ (voir Fig. 2) :

- d'un enroulement global unique permettant une réalisation aisée et un facteur de remplissage meilleur ;

- d'aimants alternés fixes (statoriques) d'aimantation radiale ;

- d'un rotor denté passif ne comportant ni bobinage, ni aimant ; permettant ainsi des vitesses de rotation élevées ;

- d'un circuit de retour de flux composé de deux culasses magnétiques extérieure et intérieure.

La circulation du flux dans ce type de structure s'effectuant dans les deux plans, le circuit magnétique ne peut être facilement feuilleté. Pour cette raison, nous avons réalisé celui-ci en matériau magnétique massif composé de poudre de fer. Ceci permet, pour les faibles encombrements, une réalisation moulée particulièrement aisée en grande série ainsi qu'un fonctionnement à hautes vitesses (> $10000 \mathrm{tr} / \mathrm{min}$ ) sans pertes fer excessives. En contre partie, la perméabilité 


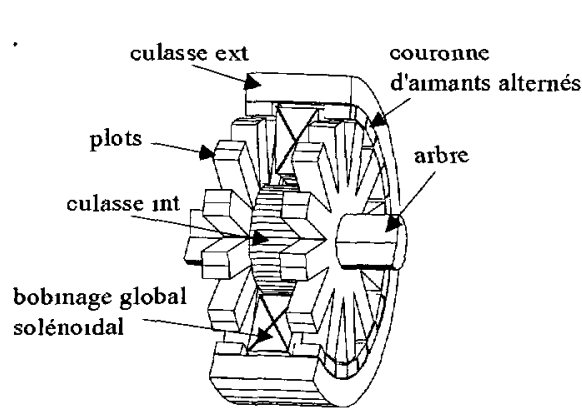

a)

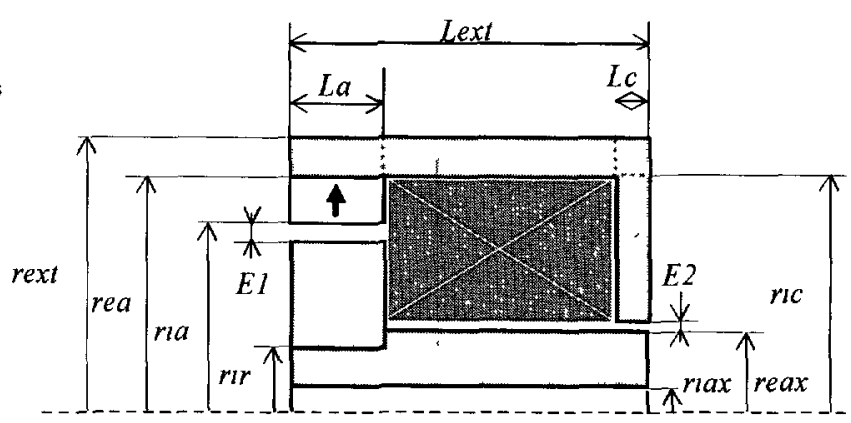

b)

Fig. 2. - Machine monophasée à aimants alternés statoriques. a) Vue en perspective d'une structure à deux partıes actıves. b) Coupe longitudınale d'une structure à une partie actıve.

[Single phase machine with stator alternated magnet. a) perspective view of a structure with two active parts, b) longitudinal cross section of structure with one actıve part.]

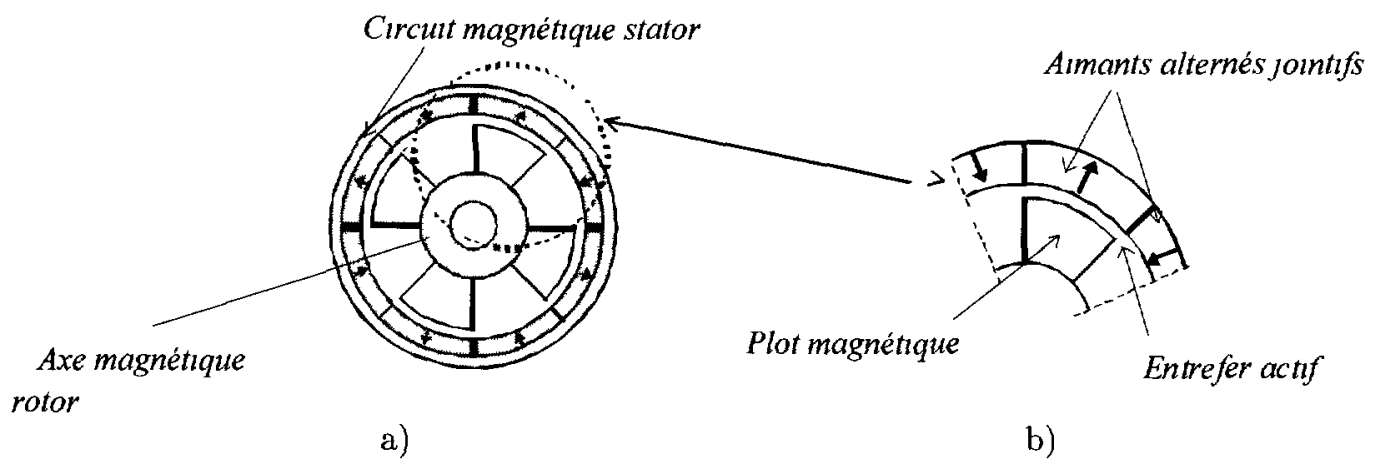

Fig. 3. - Zone active de la machine (a) et cellule élémentaire (b)

[a) Machine's active part, b) elementary pattern.]

de ce matériau est faible ce qui nécessite notamment de ne pas négliger la circulation du champ dans le fer.

De précédents travaux ont montré qu'il était possible, sous certaines conditions, de ramener l'étude d'une machine à bobinage global à celle d'une étude élémentaire cellulaire [5,7].

Nous avons appliqué ce principe pour modéliser la zone active de la machine (Fig. 2b), composée de plusieurs cellules identiques, ou motifs élémentaires, mis en parallèle et magnétisés par la même bobine.

Par conséquent, comme l'illustre la figure 3, une machine de $N_{\mathrm{p}}$ paires de pôles sera constituée de $N_{\mathrm{p}}$ cellules élémentaires.

Chaque cellule est donc composée de deux aimants permanents jointifs à aimantation radiale alternée. Elle comporte également un plot magnétique, ou dent, séparé des aimants par un jeu mécanique, ou entrefer. Le plot, ici solidaire du rotor, est moble. Noțons que le fonctionnement serait rigoureusement identique avec une denture fixe et des aimants alternés tournants.

En dehors de cette zone, la circulation du flux est assurée par l'intermédiaire de la culasse, de la couronne arrière du stator comprenant un entrefer "inactif" et enfin par l'axe rotor traversant la bobine. Ces différentes parties constituent le cırcuıt de retour de flux. Il sera modélisé de 


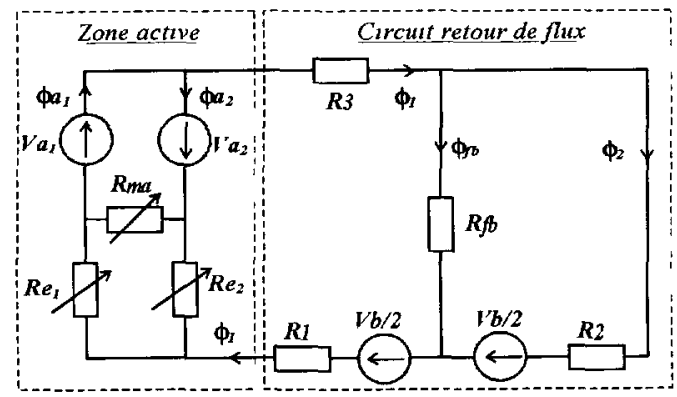

Fig. 4. - Schéma magnétique équivalent

[Equivalent magnetic circuit.]

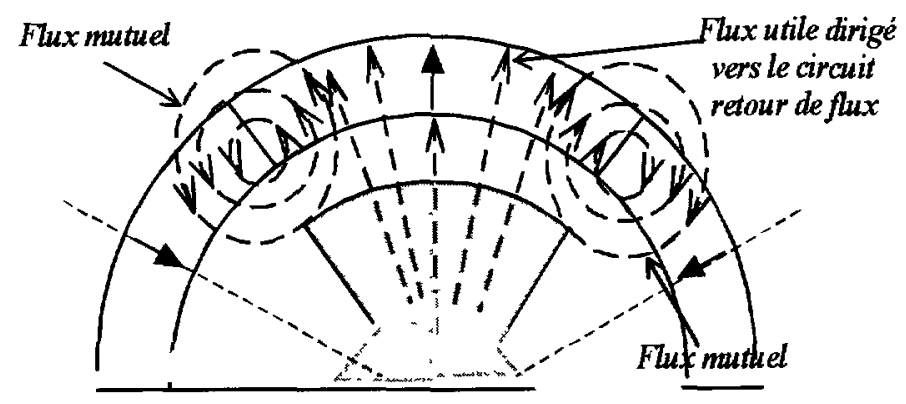

Fig. 5. - Carte de champ inducteur.

[No load field distribution.]

manière analytique.

Par ailleurs, tout au long de cette étude, nous ferons certaines hypothèses :

- le régime de fonctionnement est linéaire, la perméabilité relative $\mu_{\mathrm{rf}}$ des pièces magnétiques est faible mais constante ;

- les aimants sont jointifs, homogènes, de perméabilité constante égale à $\mu_{r a}$;

- les effets d'extrémités sont négligés. Les composantes de l'induction et du champ magnétique suivant la troisième dimension sont supposées nulles.

- précisons enfin que l'alimentation de la structure est supposée être en créneaux de courant parfaits en phase avec la force électromotrice.

2.2. SCHÉMA MAGNÉTIQUE ÉQUIVALENT - - Le schéma magnétique équivalent de la structure $2 \mathrm{~b}$ est donné par la figure 4. Notons la présence d'une réluctance placée entre les aimants, notée $R_{\mathrm{ma}}$, et d'une réluctance $R_{\mathrm{fb}}$ symbolisant respectivement la mutuelle entre aimants et les fuites magnétiques dans l'espace bobinable.

Les éléments du schéma représentant la zone active sont les suivants :

- $V_{\mathrm{a}_{1}}, V_{\mathrm{a}_{2}}$, calculés avec la relation classique (1), qui symbolisent la force magnétomotrice de chaque secteur d'aimant (de perméabilité relative $\mu_{\mathrm{ra}}$ et d'induction rémanente $B_{\mathrm{r}}$ ) 


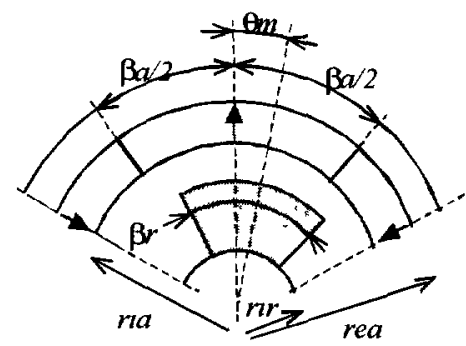

a)

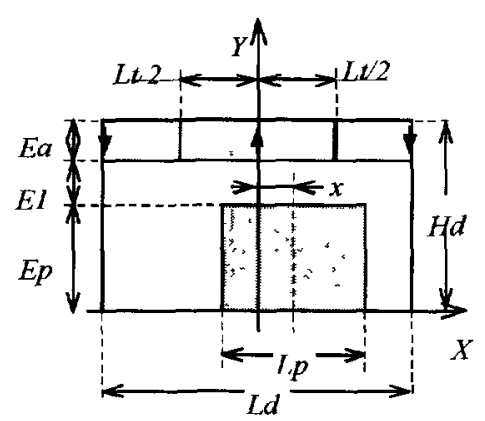

b)

Fig. 6. - Transformation de la cellule réelle (a) dans un schéma développé (b)

[Real elementary pattern transformation (a) to a developed one (b).]

présent au sein de la cellule élémentaire.

$$
V_{\mathrm{a}}=\frac{B_{\mathrm{r}}}{\mu_{0} \mu_{\mathrm{ra}}}\left(r_{\mathrm{ea}}-r_{\mathrm{ia}}\right)
$$

Dans cette expression, $r_{\mathrm{ea}}$ et $r_{1 \mathrm{a}}$ sont respectivement les rayons extérieur et intérieur des aimants.

- $R_{\mathrm{e}_{1}}, R_{\mathrm{e}_{2}}, R_{\mathrm{ma}}$, représentent des réluctances dont les valeurs varient en fonction de la position relative rotor / stator. Ces trois réluctances sont relatives respectivement au flux utile et au flux mutuel inter-aimants tel que le montre la figure 5.

Le circuit de retour est modélisé à l'aide des éléments suivants :

- les réluctances $R_{1}, R_{2}, R_{3}$ représentent chacune une zone du circuit de retour de flux,

- la réluctance $R_{\mathrm{fb}}$ symbolise, quant à elle, l'emplacement occupé par la bobine. Elle nous permet ainsi de prendre en compte de façon globale l'effet du flux de fuites de la bobine.

- la force magnétomotrice créée par la bobine est séparée en deux valeurs, notées $V_{\mathrm{b}} / 2$, chacune égale à $n i / 2$.

Les réluctances, $R_{1}, R_{2}, R_{3}$ et $R_{\mathrm{fb}}$, ne dépendent pas de la position du rotor. Par conséquent, nous les calculerons analytiquement à partir des relations classiques en fonction des différents paramètres géométriques de la machine.

\subsection{ModÉLisation de LA zone aCtive}

2.3.1. Domaine de calcul normalisé. - La cellule élémentaire réelle doit être dans un premier temps représentée dans un schéma développé correspondant à un des motifs fondamentaux étudiés dans [5]. La figure 6 illustre cette transformation. La profondeur de la cellule ainsi définie est égale à la longueur axiale des aimants et vaut $L_{\mathrm{a}}$. 


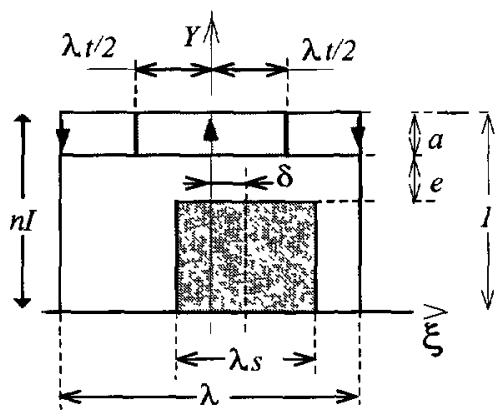

Fig 7. - Cellule élémentaire normalisée.

[Normalised elementary pattern]

Nous pouvons donc définir les paramètres de la cellule développée de la manière suivante :

$L_{\mathrm{d}}=\frac{2 \pi}{N_{\mathrm{p}}} r_{\text {moy }} \quad:$ largeur de la cellule développée,

$L_{\mathrm{t}}=r_{\text {moy }} \beta_{\mathrm{a}} \quad$ : largeur des aimants,

$L_{\mathrm{p}}=r_{\mathrm{moy}} \beta_{\mathrm{r}} \quad$ : largeur du plot,

$E_{\mathrm{a}}=\left(r_{\mathrm{ea}}-r_{\mathrm{aa}}\right) \quad$ : hauteur des aimants,

$E_{\mathrm{p}}=\left(r_{\mathrm{aa}}-E_{1}\right)-r_{\mathrm{1r}} \quad:$ hauteur du plot, avec $E_{1}$ entrefer minimal,

$H_{\mathrm{d}}=\left(E_{\mathrm{a}}+E_{\mathrm{p}}+E_{1}\right) \quad$ : hauteur de la cellule

Où

$$
r_{\text {moy }}=\left(\frac{r_{\text {ea }}+r_{\text {II }}}{2}\right)
$$

La cellule élémentaire prise en compte lors des calculs est, quant à elle, définie à l'aide d'un jeu réduit de paramètres sans dimension. Ceci permet un traitement informatique plus aisé et offre la possibilité de généraliser les résultats.

Cette seconde transformation nous permet d'obtenir une cellule élémentaire normalisée, présentée à la figure 7 , dont la hauteur est égale à l'unité.

Dans ce domaine normalisé, dont la profondeur est égale à l'unité, toutes les dimensions sont rapportées à la hauteur $H_{\mathrm{d}}$ d'une cellule. Nous obtenons alors les paramètres normalisés suivants :

$\lambda=L_{\mathrm{d}} / H_{\mathrm{d}} \quad$ : pas dentaire réduit ou facteur d'allongement du domaine,

$a=E_{\mathrm{a}} / H_{\mathrm{d}} \quad:$ hauteur réduite des aimants,

$e=E_{1} / H_{\mathrm{d}} \quad$ : entrefer actif réduit,

$s=L_{\mathrm{p}} / L_{\mathrm{d}} \quad$ : largeur angulaire normalisée des dents rotoriques,

$t=L_{\mathrm{t}} / L_{\mathrm{d}} \quad$ : taux de remplissage des aimants (pour des aimants jointifs : $t=0,5$ ).

En désignant par $\theta_{\mathrm{e}}=\theta_{\mathrm{m}} / N_{\mathrm{p}}$ la position électrique du rotor par rapport au stator, la position réelle de ce dernier dans un schéma développé est donnée par l'équation (4). Un point quelconque dans la zone active est définie par ses coordonnées linéaires $Y$ et $X$. L'abscisse $X$, pour une position rotorique donnée, est lié à l'abscisse angulaire $\theta$ de ce point par l'équation (5).

$$
\begin{aligned}
x & =\frac{r_{\mathrm{moy}}}{N_{\mathrm{p}}} \theta_{\mathrm{e}} \\
X & =\frac{r_{\mathrm{moy}}}{N_{\mathrm{p}}} \theta
\end{aligned}
$$


D'où, dans un domaine normalisé :

$\xi=\lambda \frac{\theta_{\mathrm{e}}}{2 \pi}:$ abscisse linéaire réduite

$\delta=\frac{\theta_{\mathrm{e}}}{2 \pi}:$ position rotorique normalisée.

Rappelons que la zone active, et donc chaque cellule élémentaire, est magnétisée par une force magnétomotrice ni. La cellule normalisée est, quant à elle, soumise à une force magnétomotrice normalısée $n I=n \imath / H_{\mathrm{d}}$, qui correspond donc à la valeur moyenne du champ magnétique dans la cellule.

D'autre part, dans notre modèle, le flux utile de la zone active dirigé dans le circuit de retour, noté $\phi_{1}$ représente la somme des flux de chaque cellule élémentaire, $\phi_{\mathrm{c}}$. Les $N_{\mathrm{p}}$ cellules étant toutes identiques, on obtient.

$$
\phi_{1}=N_{\mathrm{p}} \phi_{\mathrm{c}}
$$

2.3.2. Calcul de la perméance utile vue de l'aimant central $\left(P_{\mathrm{e} 1}\right)$. - La valeur de $P_{\mathrm{e} 1}$ varie en fonction de la position qu'occupe le rotor par rapport au stator. Dans un premier temps, nous la calculons pour $\delta=0$, c'est-à-dire lorsque les axes de l'aimant central et du plot sont confondus

Pour une cellule élémentaire réelle, la perméance s'écrit par définition .

$$
P_{\mathrm{e}}=\frac{\phi_{1}}{n \imath}=N_{\mathrm{p}} \frac{\phi_{\mathrm{c}}}{n \imath}
$$

En utilisant les paramètres réduits définis précédemment et sachant que la profondeur de la cellule élémentaire vaut $L_{\mathrm{a}}$, nous obtenons :

$$
P_{\mathrm{e}_{1}}(\delta=0)=N_{\mathrm{p}} \frac{L_{\mathrm{a}}}{n I} B_{\imath_{\max }} \int_{-\lambda t / 2}^{+\lambda t / 2} \bar{b}_{\imath}(\xi) \mathrm{d} \xi
$$

$\bar{b}_{\imath}(\xi)$ représente, pour une position du rotor donnée, la répartıtion de l'induction réduite engendrée par les ampères-tours normalisés seuls nI et moyennée sur toute la hauteur de l'aumant. Sa valeur est donnée par:

$$
\bar{b}_{\imath}(\xi)=\frac{1}{a} \int_{1-a}^{a} b_{2}(\xi, y) \mathrm{d} y
$$

Avec :

$$
\bar{b}_{\imath}(\xi)=\frac{\bar{B}_{\imath}(\xi)}{B_{\imath_{\max }}} \quad 0 \leq \bar{b}_{1}(\xi) \leq 1 .
$$

$B_{\imath_{\max }}$ représente l'induction maximale due aux ampères-tours seuls. Sa valeur est évaluée à partir d'un calcul analytique en appliquant le théorème d'Ampère à la cellule représentée à la figure 7. Cette valeur est calculée en considérant une ligne de champ traversant la couche d'aimants, non polarisés, de perméabilité relative $\mu_{\mathrm{ra}}$, la dent de perméabilité relative $\mu_{\mathrm{rf}}$ et l'entrefer.

$$
B_{\imath_{\max }}=\frac{\mu_{0} n I}{\left[\frac{1-a-e}{\mu_{\mathrm{rf}}}+e+\frac{a}{\mu_{\mathrm{ra}}}\right]} .
$$

Nous définissons une perméance élémentarre pour $\delta=0, \chi_{\mathrm{e}}(\delta=0)$ dont la valeur, donnée par l'équation (11), est uniquement tributaire de la répartition de l'induction réduite due aux 


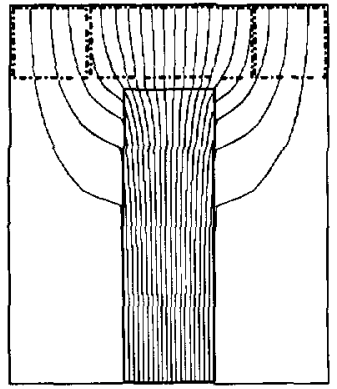

a)

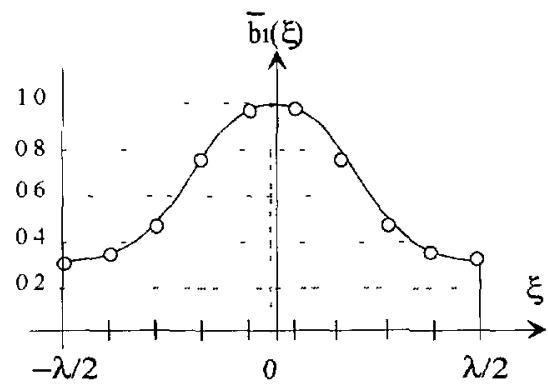

b)

Fig. 8. - Carte du champ dû aux ampères-tours (bobinage seul) (a) et répartition de l’induction réduite en fonction de l'abscisse lınéaire normalisée (b).

[Magnetic distrıbution due to ampere-turns (with no magnetised magnet) (a) and normalised magnetıc induction as function of the normalised linear abscissa (b).]

ampères-tours seuls. La figure 8 présente un exemple de tracé

$$
\chi_{\mathrm{e}}(\delta=0)=\int_{-\lambda t / 2}^{+\lambda t / 2} \bar{b}_{1}(\xi) \mathrm{d} \xi
$$

Afin de faciliter les calculs ultérieurs (f.e.m., couples, .. ), la répartition spatiale réduite $\bar{b}_{\imath}(\xi)$, calculée par éléments finis, est développée en série de Fourier. La période de cette fonction est égale à $\lambda$; elle correspond à un pas électrique de la machine. D'autre part, $\bar{b}_{1}(\xi)$ est paire et symétrıque par rapport à l'axe passant par $\xi=0$.

$$
\bar{b}_{\imath}(\xi)=\frac{a_{0}}{2}+\sum_{n=1}^{\infty}\left[a_{n} \cos \left(n \frac{2 \pi}{\lambda} \xi\right)\right] \text {. }
$$

Les coefficients $a_{0}, a_{1}, a_{2}, \ldots, a_{n}$, sont calculés à partir de l'expression classique

$$
a_{n}=\frac{4}{\lambda} \int_{0}^{\lambda / 2}\left[\bar{b}_{\imath}(\xi) \cos \left(n \frac{2 \pi}{\lambda} \xi\right)\right] \mathrm{d} \xi .
$$

Ainsi, en utilisant les relations (7) et (12), l'expression de la perméance $P_{\mathrm{e}_{1}}$ à $\delta=0$, s'écrit :

$$
P_{\mathrm{e}_{1}}(\delta=0)=\frac{\mu_{0}}{\left[\frac{1-a-e}{\mu_{\mathrm{r} \mathrm{r}}}+e+\frac{a}{\mu_{\mathrm{ra}}}\right]} N_{\mathrm{p}} L_{\mathrm{a}} \lambda\left[\frac{a_{0}}{2} t+\sum_{n=1}^{\infty} \frac{a_{n}}{n \pi} \sin (n \pi t)\right] .
$$

Dans cette expression, seuls les termes $a_{0}, a_{1}, \ldots, a_{n}$ sont inconnus. Ces valeurs sont obtenues à partir d'un calcul par éléments finis en régime linéaire.

Nous savons que la perméance $P_{\mathrm{e}_{1}}$ varie selon la position qu'occupe le plot par rapport à l'aimant central. Nous venons de la calculer pour la position $\delta=0$. nous devons maintenant déterminer son expression pour une position quelconque.

Le déplacement du rotor d'une quantité $\delta$ au sein de la cellule normalisée est équivalent au déplacement des aimants de la même quantité. La perméance élémentaire toujours face à 
l'aumant central se définit alors à partir de la relation suivante où les bornes d'intégration sont fonction de $\delta$ :

$$
\chi_{\mathrm{e}}(\delta)=\int_{(-\lambda t / 2)+\delta}^{(+\lambda t / 2)+\delta} \bar{b}_{2}(\xi) \mathrm{d} \xi=\frac{a_{0}}{2} \lambda t+\sum_{n=1}^{\infty} a_{n} \frac{\lambda}{\pi n} \sin (n \pi t) \cos (2 \pi n \delta) .
$$

La variation de la perméance $P_{\mathrm{e}_{1}}$ peut être finalement exprimée sous la forme :

$$
P_{\mathrm{e}_{1}}(\delta)=\frac{\mu_{0}}{\left[\frac{1-a-e}{\mu_{\mathrm{rf}}}+e+\frac{a}{\mu_{\mathrm{ra}}}\right]} N_{\mathrm{p}} L_{\mathrm{a}} \lambda\left[\frac{a_{0}}{2} t+\sum_{n=1}^{\infty} \frac{a_{n}}{n \pi} \sin (n \pi t) \cos (2 \pi n \delta)\right] .
$$

Précisons que $\delta=0$ ou $\delta=1$ correspondent à la position de conjonction (flux inducteur maximal) et $\delta=1 / 4$ représente la position de flux inducteur nul (ı.e. position de court-circuit des aimants).

2.3.3. Calcul de la perméance utile d'entrefer vue des aimants latéraux $\left(P_{e_{2}}\right)$. - Dans une position de flux inducteur maximal, on peut considérer que l'aimant en conjonction avec le plot produit un flux utile alors que ses voisins produisent un flux antagoniste fonction de la perméance $P_{e_{2}}$.

La figure 7 montre que la distance séparant l'axe de l'aimant central et celui d'un aimant latéral est égale à $\lambda / 2$.

Connaissant l'expression de la fonction $P_{\mathrm{e}_{1}}(\delta)$, nous pouvons en déduire $P_{\mathrm{e}_{2}}(\delta)$, en posant :

$$
P_{\mathrm{e}_{2}}(\delta)=P_{\mathrm{e}_{1}}(\delta+1 / 2)
$$

Par conséquent, nous obtenons :

$$
P_{\mathrm{e}_{2}}(\delta)=\frac{\mu_{0}}{\left[\frac{1-a-e}{\mu_{\mathrm{rf}}}+e+\frac{a}{\mu_{\mathrm{ra}}}\right]} N_{\mathrm{p}} L_{\mathrm{a}} \lambda\left[\frac{a_{0}}{2} t+\sum_{n=1}^{\infty} \frac{a_{n}}{n \pi} \sin (n \pi t) \cos (2 \pi n(\delta+1 / 2))\right] .
$$

Connaissant la relation liant les variables $\delta$ et $\theta_{\mathrm{m}}$ et les expressions de $P_{\mathrm{e}_{1}}(\delta)$ et $P_{\mathrm{e}_{2}}(\delta)$, nous pouvons déterminer les variations des réluctances $R_{\mathrm{e}_{1}}\left(\theta_{\mathrm{m}}\right)$ et $R_{\mathrm{e}_{2}}\left(\theta_{\mathrm{m}}\right)$ intervenant dans le schéma magnétique équivalent en utilisant les relations classiques suivantes :

$$
R_{\mathrm{e}_{1}}=\frac{1}{P_{\mathrm{e}_{1}}\left(\theta_{\mathrm{m}}\right)}
$$

et

$$
R_{\mathrm{e}_{2}}=\frac{1}{P_{\mathrm{e}_{2}}\left(\theta_{\mathrm{m}}\right)}
$$

2.3.4. Calcul de la perméance mutuelle $\left(P_{\mathrm{ma}}\right)$. - Par raison de symétrie, les valeurs de $P_{\mathrm{ma}}$ à $\delta=0$ et $\delta=1 / 2$ sont identiques. Par ailleurs, le calcul de $P_{\mathrm{ma}}$ en ces points permet de connaître les valeurs minimales et maximales de la fonction $P_{\mathrm{ma}}(\delta)$.

Dans l'objectif d'une étude paramétrique simplifée et rapide, nous avons approximé cette fonction à une sinusoïde. Dans ce cas, sa période est égale à $1 / 2$ puisque $P_{\mathrm{ma}}(\delta=0)$ est égal à $P_{\mathrm{ma}}(\delta=1 / 2)$, et ses valeurs oscillent autour d'une valeur moyenne notée $\bar{P}_{\mathrm{ma}}$ et qui vaut :

$$
\bar{P}_{\mathrm{ma}}=\frac{P_{\mathrm{ma}}(\delta=0)+P_{\mathrm{ma}}(\delta=1 / 4)}{2} .
$$




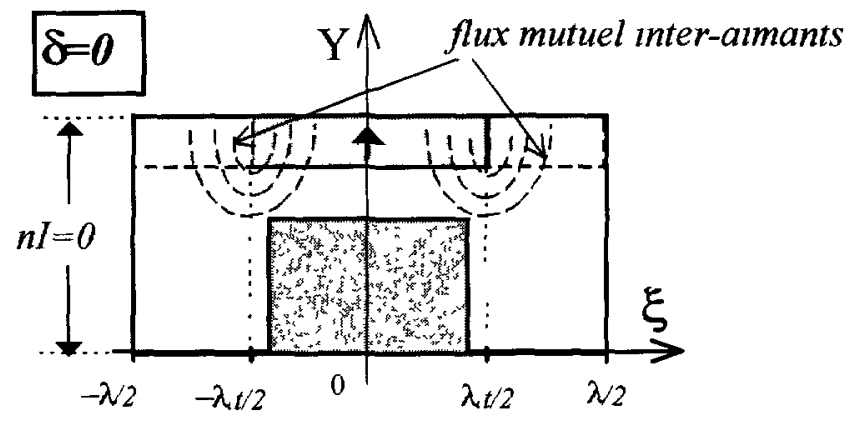

Fig 9. - Cellule normalisée avec le plot face à l'aimant central quı est le seul à ètre polarisé (position de conjonction)

[Normalised pattern in conjunction position with the only central magnet to be polarised.]

Par conséquent, selon l'hypothèse précitée, la fonction $P_{\mathrm{ma}}(\delta)$ s'écrit :

$$
P_{\mathrm{ma}}(\delta)=\vec{P}_{\mathrm{ma}}+\left(\frac{P_{\mathrm{ma}}(\delta=0)-P_{\mathrm{ma}}(\delta=1 / 4)}{2}\right) \cos (4 \pi \delta) .
$$

Connaissant la relation liant les variables $\delta$ et $\theta_{\mathrm{m}}$, nous pouvons déterminer la réluctance $R_{\mathrm{ma}}$ utilisée dans notre schéma, en posant :

$$
R_{\mathrm{ma}}\left(\theta_{\mathrm{m}}\right)=\frac{1}{P_{\mathrm{ma}}\left(\theta_{\mathrm{m}}\right)}
$$

La détermination de la perméance mutuelle inter-aimants en fonction de la position du rotor est donc tributaire du calcul de celle-ci dans les deux positions caractéristiques $\delta=0$ et $\delta=1 / 4$. Dans ce qui suit, nous décrivons la démarche utilisée.

2.3.4.1. Calcul de $P_{\mathrm{ma}}(\delta=0)$ - - Pour effectuer ces calculs, nous reprenons le formalisme développé précédemment lors du calcul de $P_{\mathrm{e}_{1}}$. Le calcul de $P_{\mathrm{ma}}$, à $\delta=0$, est effectué à partir

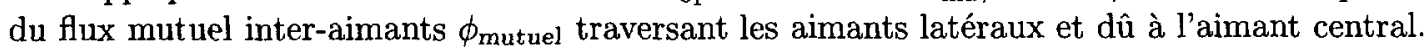
Dans cette position (voir Fig. 9), le flux est généré par l'aimant central qui est le seul à être polarisé $(n I=0)$. Pour une cellule élémentaire nous posons :

$$
P_{\mathrm{ma}}=\frac{\phi_{\text {mutuel }}}{V_{\mathrm{a}}}
$$

où $V_{\mathrm{a}}$ représente la force magnétomotrice d'un aimant (Éq. (1)).

En respectant le formalisme décrit précédemment, nous pouvons écrire, pour les $N_{\mathrm{p}}$ cellules en parallèle et pour $\delta=0$ :

$$
P_{\mathrm{ma}}(\delta=0)=\frac{N_{\mathrm{p}} \mu_{0} \mu_{\mathrm{ra}}}{B_{\mathrm{r}} E_{\mathrm{a}}} L_{\mathrm{d}} L_{\mathrm{a}} B_{\mathrm{a}_{\max }} \frac{1}{\lambda}\left[\int_{-\lambda / 2}^{-\lambda t / 2} \bar{b}_{\mathrm{a}}(\xi) \mathrm{d} \xi+\int_{\lambda \mathrm{t} / 2}^{\lambda / 2} b_{\mathrm{a}}(\xi) \mathrm{d} \xi\right] .
$$

$\bar{b}_{\mathrm{a}}(\xi)$ représente, pour une position du rotor donnée, la répartition de l'induction réduite engendrée par les aimants seuls. Sa valeur est donnée par :

$$
\bar{b}_{\mathrm{a}}(\xi)=\frac{1}{a} \int_{1-a}^{a} b_{\mathrm{a}}(\xi, y) \mathrm{d} y .
$$




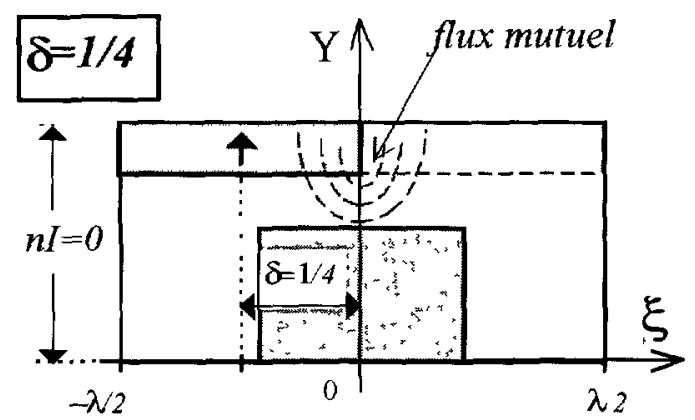

Fig 10. - Cellule normalısée où seul l'aumant de gauche est polarisé et pour $\delta=1 / 4$ (position de flux inducteur nul).

[Normalised pattern for $\delta=1 / 4$ with the only left magnet to be polarised]

Avec .

$$
\bar{b}_{\mathrm{a}}(\xi)=\frac{\bar{B}_{\mathrm{a}}(\xi)}{B_{\mathrm{r}}} \quad 0 \leq \bar{b}_{\mathrm{a}}(\xi) \leq 1
$$

où $B_{\mathrm{r}}$ représente l'induction rémanente des aimants.

En utilisant les paramètres géométriques réduits, l'expression (25) de la perméance mutuelle des aimants à $\delta=0$ s'écrit .

$$
P_{\mathrm{ma}}(\delta=0)=N_{\mathrm{p}} \frac{\mu_{0} \mu_{\mathrm{ra}}}{a} L_{\mathrm{a}} \chi_{\mathrm{ma}}(\delta=0)
$$

où $\chi_{\mathrm{ma}}(\delta=0)$ représente la perméance mutuelle élémentatre pour la position $\delta=0$. Son expression est donnée par :

$$
\chi_{\mathrm{ma}}(\delta=0)=\left[\int_{-\lambda / 2}^{-\lambda t / 2} \bar{b}_{\mathrm{a}}(\xi) \mathrm{d} \xi+\int_{\lambda t / 2}^{\lambda / 2} \bar{b}_{\mathrm{a}}(\xi) \mathrm{d} \xi\right]
$$

Comme pour $P_{\mathrm{e}_{1}}$ et $P_{\mathrm{e}_{2}}$ la détermination de $P_{\mathrm{ma}}(\delta=0)$ s'effectue par le calcul de $\bar{b}_{\mathrm{a}}(\xi)$ par éléments finis dans un domanne normalisé où $n I=0$.

2.3.4.2. Calcul de $P_{\mathrm{ma}}(\delta=1 / 4)$. - Pour cette position, le formalisme précédent est identique. Cette position, où les aimants sont court-circuités par le plot, est illustrée par la figure 10.

À $\delta=1 / 4$, la perméance mutuelle des armants est définie par l'expression suivante :

$$
P_{\mathrm{ma}}(\delta=1 / 4)=N_{\mathrm{p}} \frac{\mu_{0} \mu_{\mathrm{ra}}}{a} L_{\mathrm{a}} \chi_{\mathrm{ma}}(\delta=1 / 4)
$$

où $\chi_{\mathrm{ma}}(\delta=1 / 4)=\left[\int_{0}^{\lambda / 2} \bar{b}_{\mathrm{a}}(\xi) \mathrm{d} \xi\right]$ représente la perméance mutuelle élémentazre pour la position $\delta=1 / 4$.

Le calcul des éléments de la cellule élémentaire (zone active) par éléments finis, ainsi que les valeurs des réluctances du circuit de retour déterminées analytıquement en utilisant des relations classiques permet un dimensionnement électromagnétique complet de la machine à partir du schéma magnétique équivalent. 


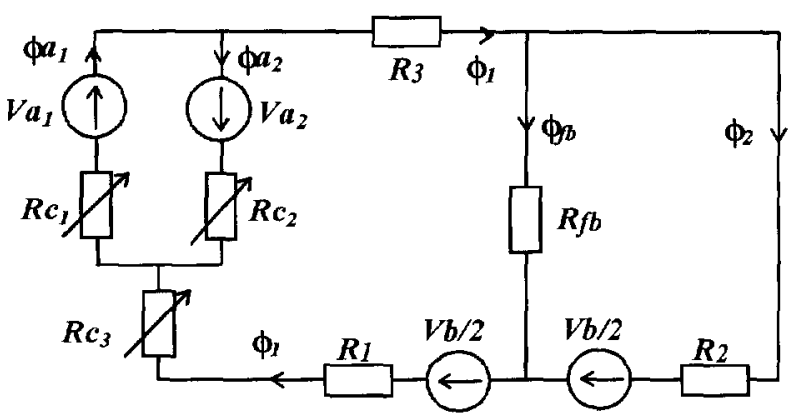

Fig. 11. - Schéma magnétique équivalent après transformation triangle-étoile.

[Equivalent magnetic circuit after $\Delta / Y$ transformation.]

\subsection{Calcul des PRINCIPAles CaractéRISTiques de la maChine}

2.4.1. Équations des différents flux. - Dans le schéma magnétique initial, présenté à la figure 4 , les réluctances $R_{\mathrm{e}_{1}}, R_{\mathrm{e}_{2}}$ et $R_{\mathrm{ma}}$ forment un circuit triangle. Afin de faciliter la mise en place des différentes expressions issues du schéma, nous transformons ce réseau triangle en étoile en appliquant le théorème de Kennely. La nouvelle structure qui en résulte est présentée à la figure 11 .

Le schéma équivalent est conçu de telle manière que dans une moitié de la bobine chaque spire est traversée par $\phi_{1}$ et dans l'autre par $\phi_{2}$.

Par conséquent, nous posons que le flux par spire traversant la bobine complète vaut :

$$
\phi_{\mathrm{b}}=\frac{\phi_{1}+\phi_{2}}{2}=\frac{\phi_{\mathrm{a}_{1}}-\phi_{\mathrm{a}_{2}}+\phi_{2}}{2} .
$$

En tenant compte de cette dernière relation, la résolution du système d'équation issu du schéma magnétique équivalent nous permet d'établir les formules analytiques avec lesquelles nous calculons les valeurs des flux $\phi_{\mathrm{a}_{1}}$ et $\phi_{\mathrm{a}_{2}}$ traversant les $N_{\mathrm{p}}$ aimants nord (indice 1) et les $N_{\mathrm{p}}$ aimants sud (indice 2), ainsı que celle du flux $\phi_{\mathrm{b}}$ traversant chaque spire de la bobine. Après résolution du système d'équation, nous obtenons ainsi .

$$
\left\{\begin{array}{l}
\phi_{\mathrm{a}_{1}}=a_{1} V_{\mathrm{a}_{1}}+m_{\mathrm{a}_{1} \mathrm{a}_{2}} V_{\mathrm{a}_{2}}+m_{\mathrm{a}_{1} \mathrm{~b}} V_{\mathrm{b}} \\
\phi_{\mathrm{a}_{2}}=m_{\mathrm{a}_{1} \mathrm{a}_{2}} V_{\mathrm{a}_{2}}+a_{2} V_{\mathrm{a}_{2}}+m_{\mathrm{a}_{2} \mathrm{~b}} V_{\mathrm{b}} \\
\phi_{\mathrm{b}}=m_{\mathrm{a}_{1} \mathrm{~b}} V_{\mathrm{a}_{1}}+m_{\mathrm{a}_{2} \mathrm{~b}} V_{\mathrm{a}_{2}}+b V_{\mathrm{b}}
\end{array}\right.
$$

Les termes $a_{1}, a_{2}, b, m_{\mathrm{a}_{1} \mathrm{~b}}, m_{\mathrm{a}_{2} \mathrm{~b}}$ et $m_{\mathrm{a}_{1} \mathrm{a}_{2}}$ sont homogènes à des perméances (voir annexe). Leurs valeurs dépendent à la fois des réluctances de la zone active (calculées par éléments finis à partir des perméances élémentaires) et de celles du circuit de retour de flux.

2.4 2. Expressions des différents couples générés par la machine. - Rappelons que pour réaliser cette étude, nous nous sommes placés dans l'hypothèse d'un milieu non saturé, caractérisé par des perméabilités constantes.

Compte tenu de ces hypothèses, le couple électromagnétique $C_{\mathrm{e}}$ produit par la machine peut être aisément calculé à partir de la variation de la coénergie magnétique $W_{\text {mag }}^{\prime}$ [1] .

Ainsi, à partir des équations des flux (32), le couple électromagnétique s'écrit dans le cas où $V_{\mathrm{a}_{1}}=V_{\mathrm{a}_{2}}=V_{\mathrm{a}}$ :

$$
C_{\mathrm{e}}\left(\theta_{\mathrm{m}}\right)=\frac{\partial W_{\mathrm{mag}}^{\prime}}{\partial \theta_{\mathrm{m}}}=\frac{1}{2} V_{\mathrm{a}}^{2}\left[\frac{\partial a_{1}}{\partial \theta_{\mathrm{m}}}+\frac{\partial a_{2}}{\partial \theta_{\mathrm{m}}}+2 \frac{\partial m_{\mathrm{a}_{1} \mathrm{a}_{2}}}{\partial \theta_{\mathrm{m}}}\right]+\frac{1}{2} V_{\mathrm{b}}^{2}\left[\frac{\partial b}{\partial \theta_{\mathrm{m}}}\right]
$$




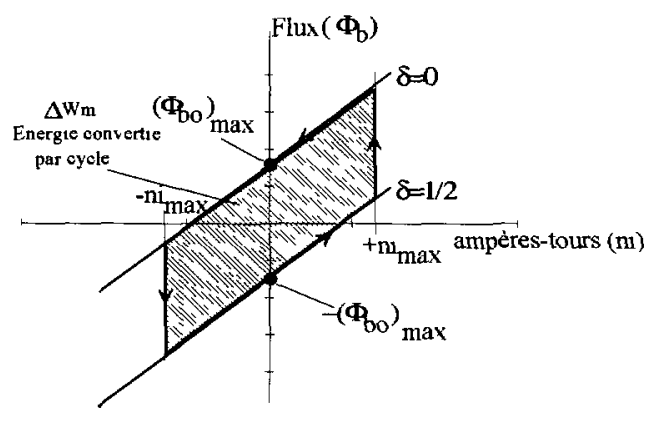

Fig. 12. - Caractéristiques magnétıques extrêmes et cycle énergétique de conversion dans le cas d'une alimentation en créneaux de courant

[Energetic strocke in ideal square current supply case ]

$$
+V_{\mathrm{a}} V_{\mathrm{b}}\left[\frac{\partial m_{\mathrm{a}_{1} \mathrm{~b}}}{\partial \theta_{\mathrm{m}}}+\frac{\partial m_{\mathrm{a}_{2} \mathrm{~b}}}{\partial \theta_{\mathrm{m}}}\right]
$$

Cette expression met en évidence trois termes, correspondants :

- au couple de détente, proportionnel au carré de la force magnétomotrice des aimants. Il est produit par les aimants seuls lorsque le moteur n'est pas alimenté (premier terme).

- au couple réluctant, proportionnel au carré de la force magnétomotrice de la bobine. Rappelons que dans le cas de notre machine, cette composante est nulle. En effet, le terme $b$ reste constant quelle que soit la position du rotor (deuxième terme).

- au couple hybride, proportionnel au produit de la force magnétomotrice des aimants et de la bobine. Il est donc produit par l'interaction de ces deux éléments (troisième terme).

Pour un courant en créneaux parfaits et dans l'hypothèse d'un régime linéaire et en négligeant les pertes (fer et mécaniques), on montre [5] que le couple électromagnétique moyen $\bar{C}_{\mathrm{e}}$ est proportionnel au nombre de paires de pôles $N_{\mathrm{p}}$, à la force magnétomotrice de la bobine $V_{\mathrm{b}}=n i_{\max }$ et au flux inducteur maximal $\phi_{\mathrm{bo}}{ }_{\max }$.

$$
\bar{C}_{\mathrm{e}}=\frac{2 N_{\mathrm{p}}}{\pi} \phi_{\mathrm{bo} \max } n \imath_{\max }
$$

Pour une position du rotor donnée, le flux inducteur $\phi_{\mathrm{bo}}$ se calcule à partir de l'expression (32) dans laquelle le terme $V_{b}$ est nul. Sa valeur est donc égale à :

$$
\phi_{\mathrm{bo}}=V_{\mathrm{a}}\left[m_{\mathrm{a}_{1} \mathrm{~b}}+m_{\mathrm{a}_{2} \mathrm{~b}}\right] \text {. }
$$

Le flux inducteur traversant la bobine est maximal lorsque le plot rotorique se trouve face à un aimant. Par conséquent, connaissant les valeurs de $R_{\mathrm{e}_{1}}, R_{\mathrm{e}_{2}}$ et $R_{\mathrm{ma}}$ pour $\theta_{\mathrm{m}}=0$, nous pouvons calculer les valeurs des termes $m_{\mathrm{a}_{1} \mathrm{~b}}\left(\theta_{\mathrm{m}}=0\right)$ et $m_{\mathrm{a}_{2} \mathrm{~b}}\left(\theta_{\mathrm{m}}=0\right)$. La valeur maximale du flux inducteur peut s'écrire alors sous la forme .

$$
\phi_{\mathrm{bo}_{\max }}=V_{\mathrm{a}}\left[m_{\mathrm{a}_{1} \mathrm{~b}}\left(\theta_{\mathrm{m}}=0\right)+m_{\mathrm{a}_{2} \mathrm{~b}}\left(\theta_{\mathrm{m}}=0\right)\right] .
$$


2.4.3. Caractéristiques du bobinage. - En l'absence d'alimentation, le mouvement du rotor entraîne aux bornes du bobinage, comportant $n$ spires, l'apparition d'une force électromotrice alternative de fréquence $f$. Si le flux varie sinusoïdalement, la valeur maximale de la f.e.m. peut être déduite de la relation $(37)$ :

$$
E_{\mathrm{O}_{\max }}=2 \pi f n \phi_{\mathrm{bo}_{\max }} \text {. }
$$

La fréquence électrique $f$ est égale à $\frac{N_{\mathrm{p}} \Omega}{2 \pi}$ où $\Omega$ représente la vitesse de rotation en $\mathrm{rad} \mathrm{s}^{-1}$.

D'autre part, l'inductance de la machine ne dépend pas de la position du rotor puisque l'effet réluctant est nul. Elle est définie alors par la relation classique :

$$
L=n^{2}\left[\frac{\phi_{\mathrm{b}_{1}}}{n \imath}\right]
$$

où $\phi_{\mathrm{b}_{1}}$ est le flux par spire, créé par la bobine seule, qui traverse chacune des $n$ spires parcourues par le courant $i$. Ce flux se calcule à partir de l'expression (32) dans laquelle les termes représentant la force magnétomotrice des aimants alternés, $V_{\mathrm{a}}$, est nulle et pour une force magnétomotrice de la bobine $V_{\mathrm{b}}$ égale à $n \imath$.

Concernant les pertes Joule, celles-ci sont liées à la densité de courant et au volume des conducteurs par la relation suivante:

$$
P_{\text {Joule }}=\int_{V_{c}} \rho J_{\text {eff }}^{2} \mathrm{~d} v
$$

$\rho$ étant la résistivité du cuivre, $J_{\text {eff }}$ la valeur efficace de la densité de courant dans la bobine et $V_{\mathrm{c}}$ le volume de cuivre.

Par ailleurs, en définissant $K_{\mathrm{b}}$ comme étant le coefficıent de remplsssage de la bobıne et $S_{\mathrm{b}}$ la section de la fenêtre bobinable (avec $V_{\mathrm{c}}=K_{\mathrm{b}} S_{\mathrm{b}} L_{\mathrm{s}}$ ), on montre que les pertes Joule peuvent être calculées à partir de la relation suivante :

$$
P_{\text {Joule }}=\rho\left[\frac{\left(K_{1} n i_{\max }\right)^{2}}{K_{\mathrm{b}}}\right]\left(\frac{L_{\mathrm{s}}}{S_{\mathrm{b}}}\right)
$$

où $L_{\mathrm{s}}$ est la longueur moyenne d'une spire et $K_{1}$ est un facteur de forme qui dépend de l'allure du courant (pour une alimentation en créneaux, $K_{1}$ vaut 1 ).

Dans cette relation, le rapport $\left(L_{\mathrm{s}} / S_{\mathrm{b}}\right)$ est directement lié à la géométrie de la machine.

2.4.4. Détermination des pertes fer. - Un flux alternatif circulant dans un milieu ferromagnétique entraîne deux types de pertes :

- les pertes par hystérésis,

- les pertes par courants de Foucault.

Pour des ondes sinusoïdales et dans une plage de fréquence limitée, nous pouvons exprimer les pertes volumiques totales dans le fer (en $W / m^{3}$ ) par la relation de Stenmetz [14] :

$$
P_{\mathrm{fer}}=K_{\mathrm{fer}} f^{\alpha} B_{\mathrm{max}}^{\gamma}
$$

où $K_{\mathrm{fer}}, \alpha$ et $\gamma$ sont des coefficients propres à chaque matériau magnétique.

Pour notre application, les valeurs de ces coefficients sont déduites des essais que nous avons effectué sur un échantillon de poudre de fer (SAGEM F75), pour différents niveaux d'induction compris entre 0,05 et $0,6 \mathrm{~T}$ et des fréquences de 500 et $1000 \mathrm{~Hz}$. 


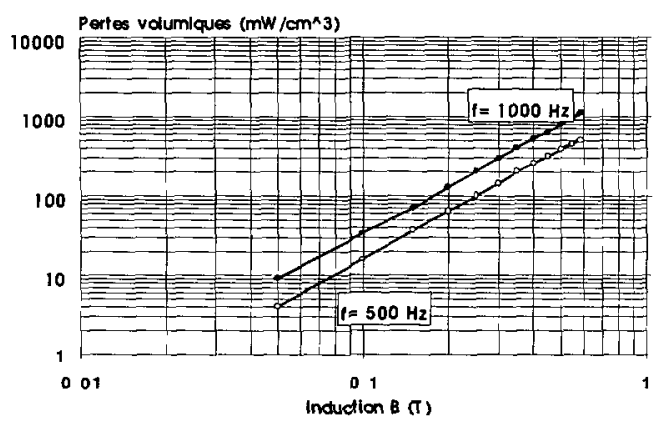

a)

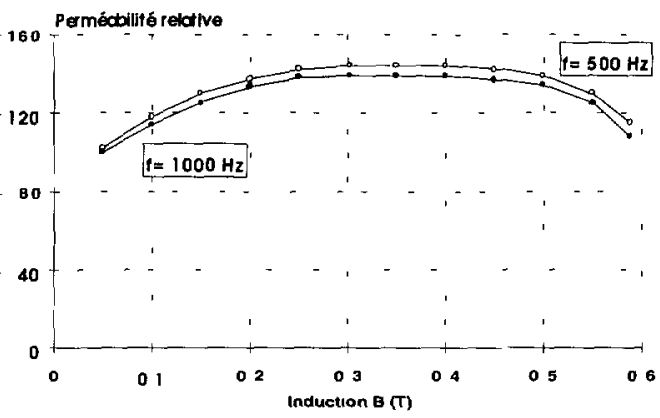

b)

Fig. 13 - Caractérıstıques magnétique mesurées de la poudre de fer ((SAGEM F75). a) Pertes volumiques, b) perméabilité relatıve.

[Magnetic characteristic of the SAGEM F75 iron powder. a) specific iron losses, b) relative permeability.]

Ces relevés, présentés à la figure 13 , nous ont notamment permis de connaître les pertes volumiques de la poudre de fer utilisée pour réaliser le circuit magnétique de la machine ainsi que les valeurs des coefficients caractéristiques : $K_{\text {fer }}=3000, \alpha=1.1$ et $\gamma=1,95$.

Nous remarquons que les pertes sont pratiquement proportionnelles à la fréquence et au carré de l'mduction Ceci confirme que les pertes dans la poudre de fer utilisée sont dominées, pour des fréquences d'utilisation inférieures à $1000 \mathrm{~Hz}$, par le phénomène d'hystérésis. Les pertes par courants de Foucault sont, quant à elles, négligeables.

Pour déterminer les pertes fer, nous devons donc connaître l'induction crête et la fréquence du flux inducteur.

Concernant l'induction crête, celle-ci est déterminée dans la zone active (cellule élémentaire) par éléments finis et analytiquement dans la région du circuit de retour de flux.

2.4.5 Évaluation de la force magnétomotrice au démarrage et en régime nommal. - Par définition, le couple mécanique fourni à la charge, ou couple utile $C_{\mathrm{u}}$, est égal au couple électromagnétique moyen $\bar{C}_{\mathrm{e}}$ auquel il faut déduire le couple engendré par les pertes mécaniques $\left(P_{\text {méca }}\right)$ et les pertes fer. La machine étant alimentée en créneaux de courant, le couple électromagnétique moyen, donné par la relation (34) ainsi que les pertes fer dépendent du courant d'alimentation. La recherche de la force magnéto-motrice nécessaire au démarrage s'effectue par conséquent par itération (dont la variable est $n i_{\text {max }}$ ) jusqu'à vérification (avec une précision donnée) de l'égalité suivante :

$$
\bar{C}_{\mathrm{e}}\left(n \imath_{\max }\right)=C_{\mathrm{u}}+\left(\frac{P_{\text {méca }}+P_{\mathrm{fer}}\left(n \imath_{\max }\right)}{\Omega}\right)
$$

Nous évaluons la force magnétomotrice nécessaire pour respecter les conditions de démarrage $n \imath_{\text {dém }}$, en remplaçant le terme $C_{\mathrm{u}}$ dans l'expression (42) par la valeur du couple de démarrage $C_{\text {dém }}$, détermınée à partir du cahıer des charges (ce couple est fonction notamment du temps de démarrage et de la tension maximale d'alimentation). De la même façon, nous évaluons la force magnétomotrice nécessaire au fonctionnement nominal $n i_{\text {nom }}$, en remplaçant cette fois-ci le terme $C_{\mathbf{u}}$ par la valeur du couple nominal en régime permanent spécifié dans le cahier des charges.

Précisons bien qu'il ne s'agit que d'une évaluation. En effet, à haute vitesse, le courant n'est 


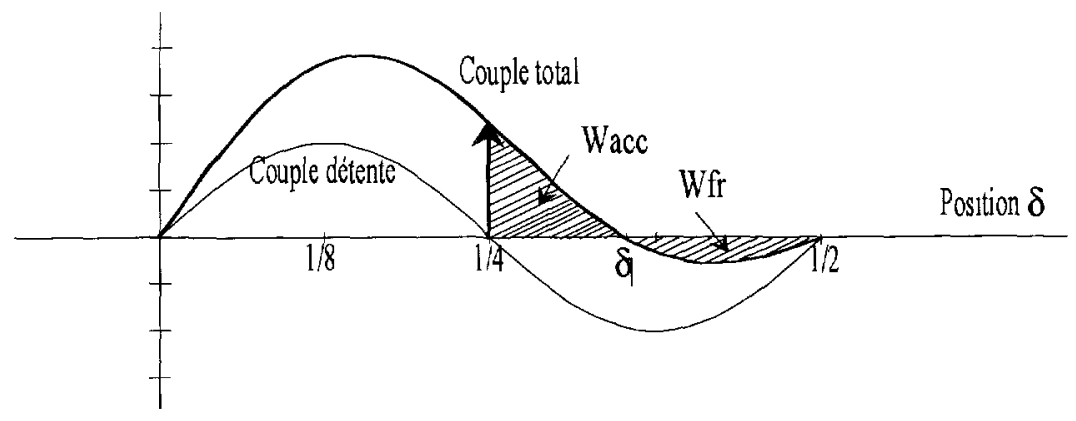

Fig 14. - Allure des couples de détente et total sur un-demi pas dentaire dans le cas d'un démarrage possible.

[Detent and total torque shapes in possible startıng case ]

plus d'allure carrée. Un calcul rigoureux nécessiterait bien entendu la prise en compte des formes d'ondes réelles du courant.

2.4.6. Vérıfication des conditions de démarrage. - Le calcul précédent nous a permis d'évaluer

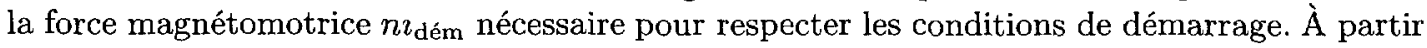
de la relation générale (33), nous pouvons déterminer et tracer, en fonction de la position du rotor $\delta$, le couple de détente $C_{\mathrm{d}}(\delta)$ et le couple hybride développé par la machine $C_{\mathrm{e}}(\delta)$, lorsque la valeur de la force magnétomotrice de la bobine est égale à $n \imath_{\text {dém }}$. La figure 14 illustre le cas d'une machine où le démarrage est possible.

Les conditions de démarrage sont fonction du couple de charge, des couples hybride et de détente, de l'inertie des parties tournantes et de la forme du courant d'alimentation Ce dernier est fonction de la structure du convertisseur, de l'amplitude et de la forme d'onde de la f.e.m. et des caractéristiques électrıques de la machine. La détermination précise des conditions de démarrage est donc complexe, nécessitant un calcul couplé : magnétique-mécanique-électrique. Cette étude est en cours au Laboratoire.

Pour notre part, nous nous intéressons aux conditions de démarrage limites, à savoir les conditions pour lesquelles, nous avons pendant le temps de démarrage :

- un courant d'alimentation supposé constant ;

- un couple de charge et une inertie négligeables ;

Dans ces conditions, nous devons au moins nous assurer que les deux conditions limites suivantes sont respectées :

- Dans une position d'équilibre, le couple électromagnétique est non nul et le couple de détente instantané présente, pour cette position, une pente négative. Compte tenu des conventions portées sur la figure 14, nous devons donc vérifier :

$$
C_{\mathrm{d}}(\delta=1 / 4)=0 \quad \text { et }\left.\quad \frac{\mathrm{d} C_{\mathrm{d}}}{\mathrm{d} \delta}\right|_{\delta=1 / 4}<0
$$

- si cette première condition est respectée, nous devons alors vérifier que l'énergie mécanique d'accélération $W_{\text {acc }}$ est supérieure à l'énergie de freinage $W_{\mathrm{fr}}$. 
Tableau I. - Caractéristıques de base du prototype.

[Basic data of the prototype.]

\begin{tabular}{|l|c|c|}
\hline Paramètres & Notation & Valeurs de base \\
\hline Nombre de spires & $n$ & 100 \\
\hline Nombre de paires de pôles & $N_{\mathrm{p}}$ & 2 \\
\hline Couple utile (Nm) & $C_{\mathrm{u}}$ & 0,01 \\
\hline Vitesse de rotation nominale (tr/min) & $N_{\mathrm{n}}$ & 15000 \\
\hline Induction rémanente (T) & $B_{\mathrm{r}}$ & 0,5 (aimants ferrites de chez Arelec) \\
\hline Perméabilité relative des aimants & $\mu_{\mathrm{ra}}$ & 1,15 \\
\hline Perméabilité du matériau magnétique & $\mu_{\mathrm{rf}}$ & 140 \\
\hline Rayon extérieur de la machine (mm) & $R_{\mathrm{ex}}$ & 20 \\
\hline Longueur aimants (mm) & $L_{\mathrm{a}}$ & 10,5 \\
\hline Largeur angulaire des aimants $\left(^{\circ}\right)$ & $\beta_{\mathrm{a}}$ & 90 \\
\hline Largeur angulaire des dents rotoriques $\left(^{\circ}\right)$ & $\beta_{\mathrm{r}}$ & 90 \\
\hline Rayon intérieur des aimants $(\mathrm{mm})$ & $R_{\mathrm{aa}}$ & 14,05 \\
\hline Rayon extérieur des aimants $(\mathrm{mm})$ & $R_{\mathrm{ea}}$ & 17,25 \\
\hline Rayon intérieur du rotor $(\mathrm{mm})$ & $R_{\mathrm{rr}}$ & 7,5 \\
\hline Jeu mécanique actıf (mm) & $E_{1}$ & 0,5 \\
\hline
\end{tabular}

Ces deux conditions peuvent êtres exprimées sous la forme d'un facteur de démarrage limite (noté $k_{\mathrm{D}}$ ) dont la valeur devra vérifier l'inégalité suivante :

$$
k_{\mathrm{D}}=\left(\left.\frac{\mathrm{d} C_{\mathrm{d}}}{\mathrm{d} \delta}\right|_{\delta=1 / 4}\right)\left|\frac{\int_{\delta=1 / 4}^{\delta_{1}} C_{\mathrm{e}}(\delta) \mathrm{d} \delta}{\int_{\delta_{1}}^{\delta=1 / 2} C_{\mathrm{e}}(\delta) \mathrm{d} \delta}\right| \ll 0 .
$$

\section{3. Étude paramétrique}

Le modèle semi-numérique présenté est d'emploi aisé et souple. Celui-ci a été utilisé pour effectuer une analyse paramétrique rapide sur l'influence des principaux paramètres géométriques et magnétiques sur les performances globales de la machine.

Les valeurs des paramètres à partir desquels cette étude a été effectuée (caractéristiques de base) sont donnés au tableau I.

3.1. InFLUENCE DE LA LARGEUR ANGULAIRE ROTORIQUE. - Les figures 15 et 16 présentent la variation des perméances calculées d'entrefer, mutuelle et des couples correspondants en fonction de la position relative du rotor pour deux valeurs de largeurs angulaires normalisées des dents rotoriques ( $s=0.3$ et $s=0.5$ ).

Ces figures montrent que la largeur angulaire rotorique est prépondérante en ce qui concerne le démarrage monophasé. L'inversion de la forme d'onde du couple de détente est obtenue ici par l'élargissement des dents rotoriques. Cette inversion est due à celle de la perméance inter-aimants. En effet, pour une largeur angulaire des dents rotoriques inférieure à celle des aimants, le flux mutuel inter-aimants est maximal dans la position de conjonction (aimant central face au plot). À l'inverse, l'élargissement des dents rotoriques au-delà des largeurs des 


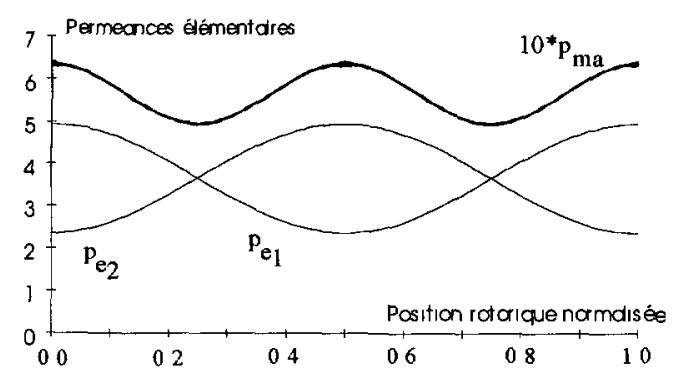

a)

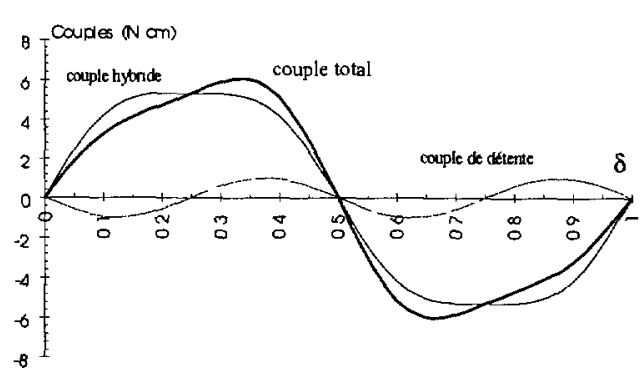

b)

Fig. 15. - Varıation des perméances de la zone active (a) et des couples générés pour $\imath=6 \mathrm{~A}$ (b) en fonction de la position relative du rotor pour une largeur angulaire rotorique normalisée de $s=0,3$.

[Permeance waveformes (a) and electromagnetic torque one (b) for $i=6 \mathrm{~A}$ and $s=0.3$.]

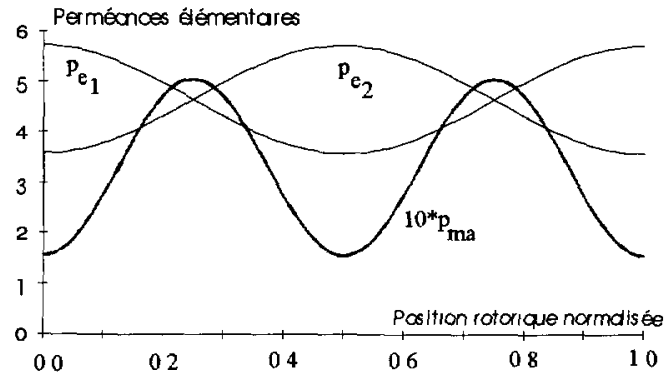

a)

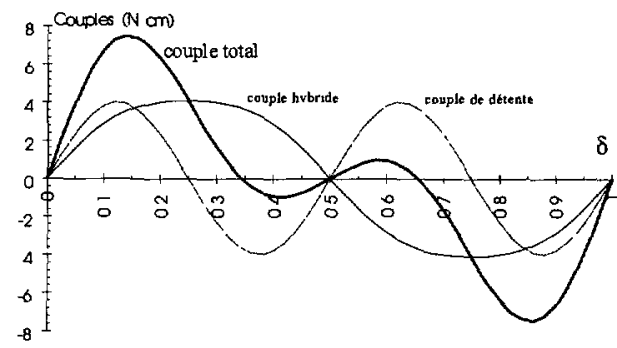

b)

Fig 16. - Variation des perméances de la zone active (a) et des couples générés pour $i=6 \mathrm{~A}$ (b) en fonction de la position relative du rotor pour une largeur angulaire rotorique normalsée de $s=0,5$.

[Permeance waveformes (a) and electromagnetic torque one (b) for $\imath=6 \mathrm{~A}$ and $s=0.5$.]

aimants permet d'obtenir un flux mutuel maximal dans la position d'opposition (position de court-circult des aimants à travers le plot).

Nous avons représenté sur la figure 17a la variation de la pente du couple de détente en position d'équilibre stable $(\delta=1 / 4)$ en fonction de la largeur angulaire des dents rotoriques $(s)$ et pour différentes épaisseurs des aimants $(a)$. Cette figure montre de manière intrinsèque et indépendante de la charge entraînée qu'il existe une valeur limite de $s$ à partir de laquelle la pente du couple de détente devient positive. La possibilité ou non d'un démarrage en monophasé est cependant mise en évidence par le facteur $k_{\mathrm{D}}$ représenté à la figure $17 \mathrm{~b}$. Par ailleurs, la largeur angulaire limite étant fonction de la hauteur des aimants, les résultats obtenus dans le cas étudié montrent que la largeur limite est de 0,32 pour des hauteurs d'aimants faibles et de 0,4 pour des aimants épais.

Cependant, l'élargissement des dents rotoriques accroît le flux antagoniste ou parasite des aimants latéraux et par conséquent entraîne la diminution du flux inducteur maximal. L'optimum de $s$ est d'environ 0,3 comme le montre la figure 18a. Ainsi, l'accroissement de $s$ à partir de sa valeur optimale entraîne :

- la hausse du courant nécessaire au fonctionnement nominal et donc l'augmentation des pertes Joule , . 


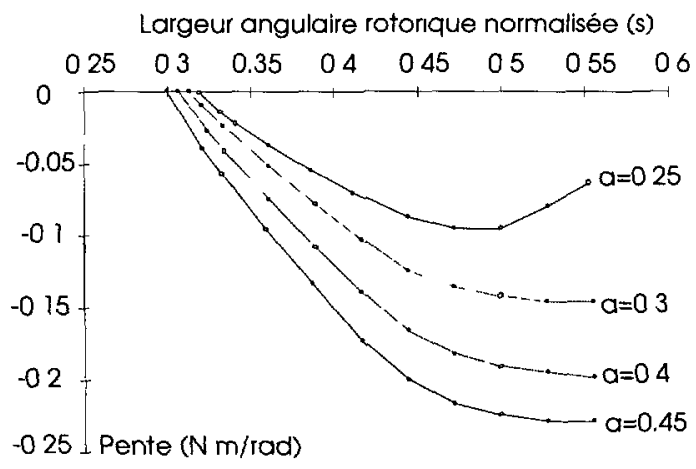

a)

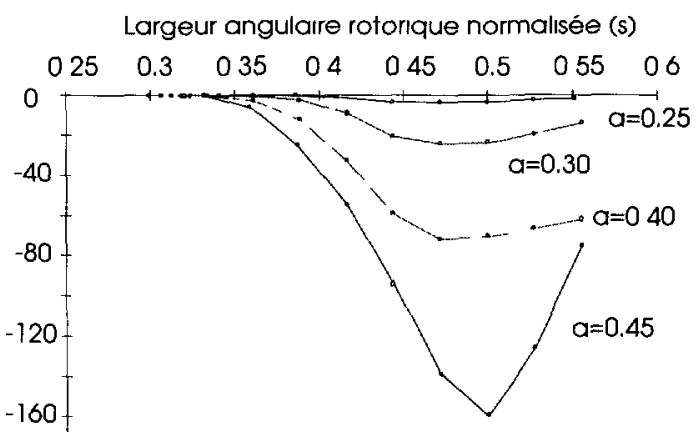

b)

Fig. 17. - Variation de la pente du couple de détente en position d'équilibre stable a) et du facteur de démarrage limite $b$ ) en fonction de la largeur normalisée angulaire des dents rotoriques

[Slope's variation of detent torque in stable position a) and limit starting factor's one as function of the normalised tooth angular width (b).]

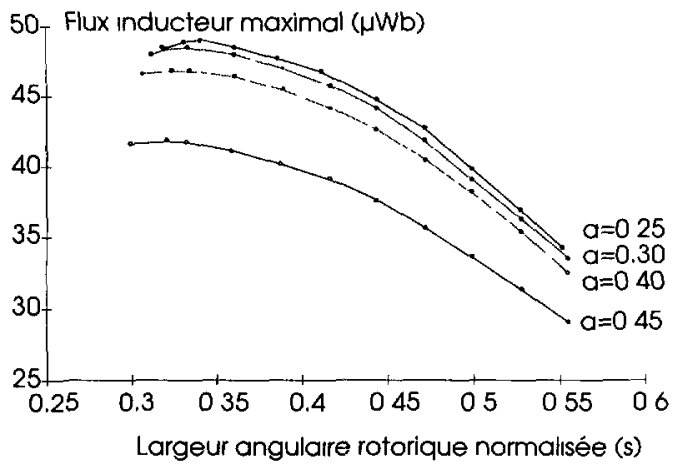

a)

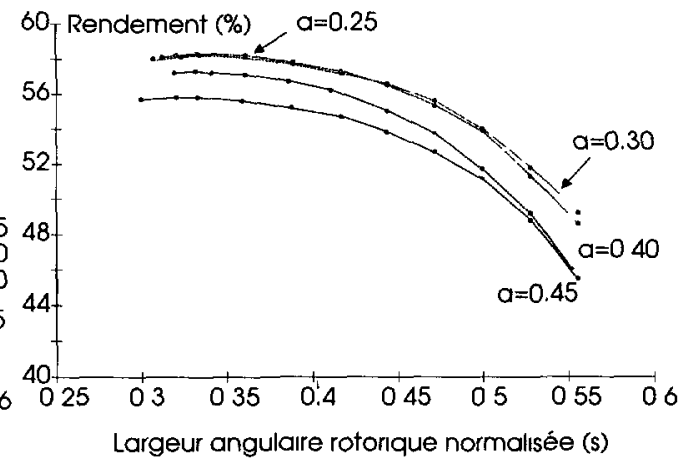

b)

Fig. 18. - Variation du flux inducteur maximal (a) et du rendement électromagnétique (b) en fonction de la largeur angulaire rotorique normalisée.

[Peak value's varıation of inductor flux (a) and electromagnetic efficiency's one as function of the normalised tooth angular width (b).]

- la diminution des pertes fer à vitesse constante due à la chute du flux inducteur.

Au delà d'une certaine largeur, la diminution des pertes fer né permet cependant pas de compenser l'augmentation des pertes Joules. Ce qui se traduit donc par la diminution du rendement électromagnétique (Fig. 18b).

3.2. INFLUENCE DE LA HAUTEUR DES AIMANTS. - La hauteur des aimants (a) est un paramètre important pour le démarrage monophasé comme on l'a vu précédemment. Son influence est prépondérante aussi dans l'évolution du flux inducteur et particulièrement à partir d'une certaine valeur.

Nous avons représenté à la figure 19a l'évolution du flux inducteur maximal en fonction de la hauteur normalisée des aimants et pour deux valeurs du jeu mécanique actif $\left(E_{1}\right)$. Ces résultats 


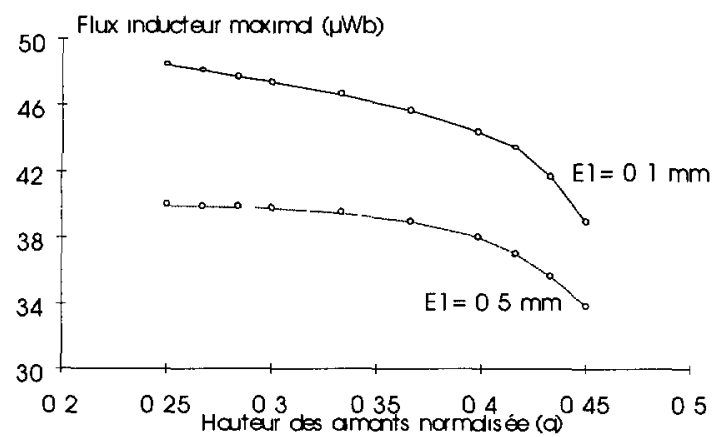

a)

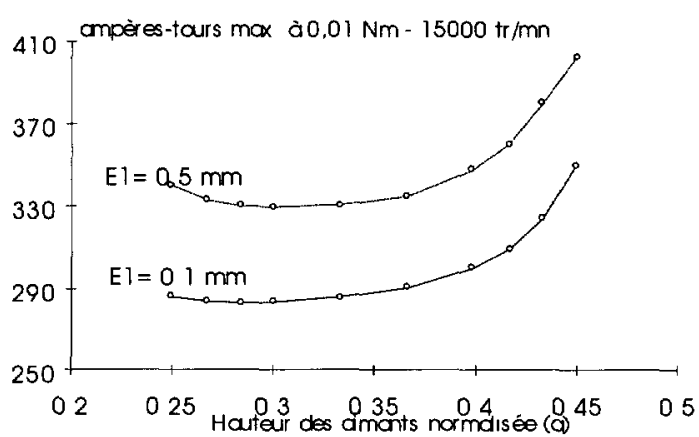

b)

Fig. 19. - Variatıon du flux inducteur maximal (a) et de la valeur nominale des ampères-tours (b) en fonction de la hauteur des aimants normalisée.

[Peak value's varlation of inductor flux (a) and ampere-turns nominal value as function of the normalised magnet's height (b).]

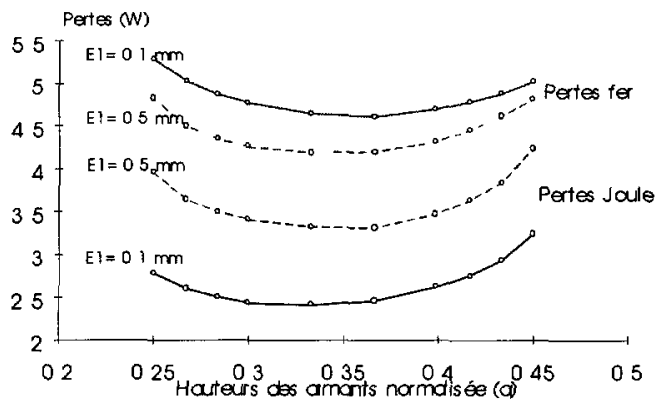

a)

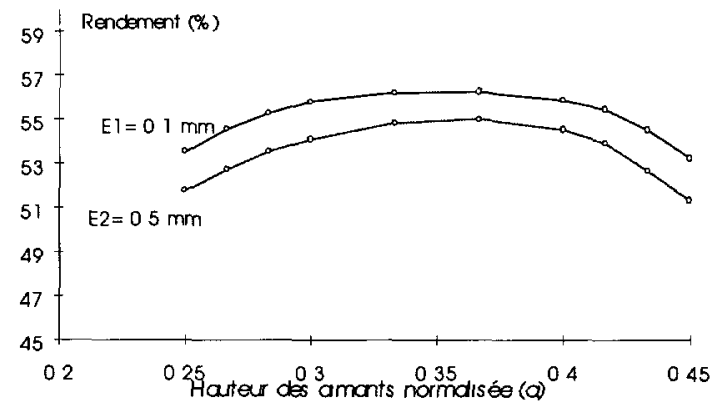

b)

Fig. 20 - Variation des pertes Joule et fer (a) et du rendement électromagnétique (b) en fonction de la hauteur des almants normalısée à $0,01 \mathrm{Nm}$ et $15000 \mathrm{tr} / \mathrm{min}$

[Variation of iron and copper losses (a) and electromagnetic efficiency (b) as function of the normalised magnet's height for $0.01 \mathrm{Nm}$ and $15000 \mathrm{rpm}$ operating point.]

obtenus montrent ainsi que l'augmentation de $a$ entraîne la diminution du flux inducteur du fait de l'accroissement du flux antagoniste généré par les aimants latéraux de polarité opposée à l'aimant central générateur de flux utile [6].

La valeur des ampères-tours nécessaires au fonctionnement nominal est obtenue à partir de la résolution itérative de l'équation (42). La diminution du flux inducteur engendre un courant d'alimentation important afin de développer le couple électromagnétique nécessaire. Cette augmentation du courant entraîne parallèlement l'accroissement des pertes Joule. Ainsi, la prise en compte simultanée des pertes Joule et fer et du couple de charge imposé dans l'évaluation du courant nominal permet de mettre en évidence une valeur minimale du courant telle que montrée par la figure 19b. Ceci se traduit par l'apparition d'une valeur optimale de $a$ pour laquelle les pertes Joule et fer (Fig. 20a) sont minimales et par conséquent le rendement correspondant est maximal (voir Fig. 20b). Dans le cas étudié, cette valeur est de 0,35. 


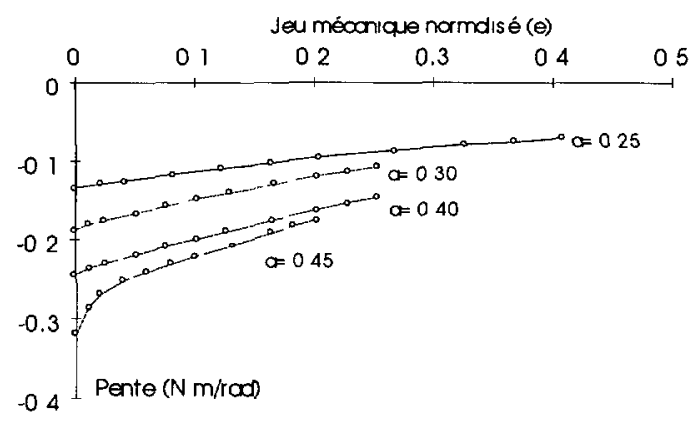

a)

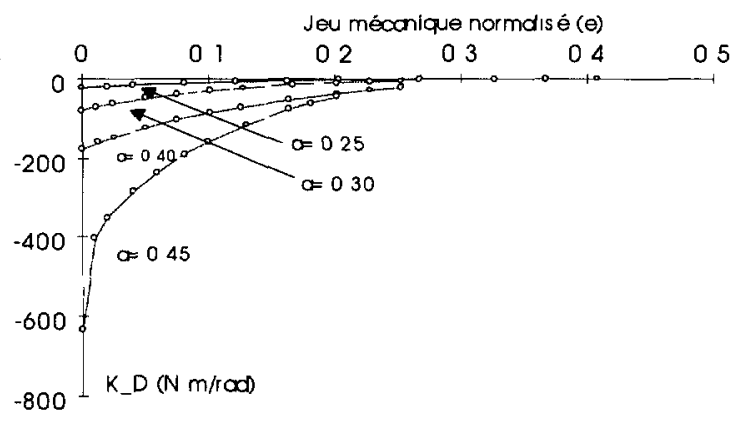

b)

Fig 21. - Variation de la pente du couple de détente en position d'équilibre stable (a) et du facteur de démarrage limite (b) en fonction du jeu mécanique actıf normalisé.

[Slope's variation of detent torque in stable position (a) and limit starting factor's one as function of the normalised actıve air-gap (b).]

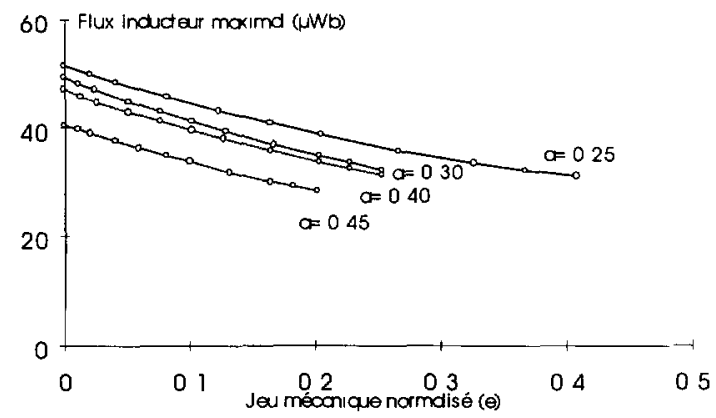

a)

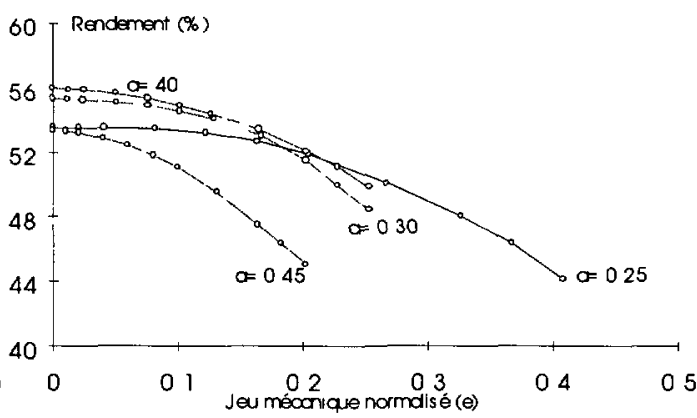

b)

Fig. 22. - Variation du flux inducteur maxımal (a) et du rendement électromagnétique (b) en fonction du jeu mécanique actif normalisé.

[Peak value's varıation of inductor flux (a) and electromagnetic efficiency's one as function of the normalised active air-gap (b).]

3.3. INFLUENCE DU JEU MÉCANIQUe ACTIF. - Deux tracés sont réalisés pour différentes valeurs du jeu mécanique normalisé (Fig. 21) correspondant à l'influence de $e$ sur les conditions de démarrage limites. Ces tracés montrent que l'augmentation du jeu mécanique normalisé entraîne la dégradation des conditions de démarrage limites. Celle-ci est d'autant plus marquée pour des hauteurs d'aimants faibles. À titre d'exemple, pour des jeux mécaniques supérieurs à 0,3 , le démarrage en monophasé n'est plus possible $\left(k_{\mathrm{D}}>0\right)$.

Par ailleurs, l'augmentation du jeu mécanique engendre la diminution du flux inducteur (Fig. 22a) du fait du court-circuit des aimants à travers ce jeu. Ceci se traduit par la dégradation du rendement électromagnétique (Fig. 22b). Cette dégradation est cependant moins marquée pour des hauteurs d'aimants faibles.

3.4. INFLUENCE DE LA PERMÉABILITÉ RELATIVE DU MATÉRIAU MAGNÉTIQUE . - Le circuit magnétique massif de la machine est réalisé à partir de poudre de fer. Celle-ci présente des pertes fer faible même à hautes fréquences. Cependant, sa perméabilité relative $\mu_{\mathrm{rf}}$ est réduite 


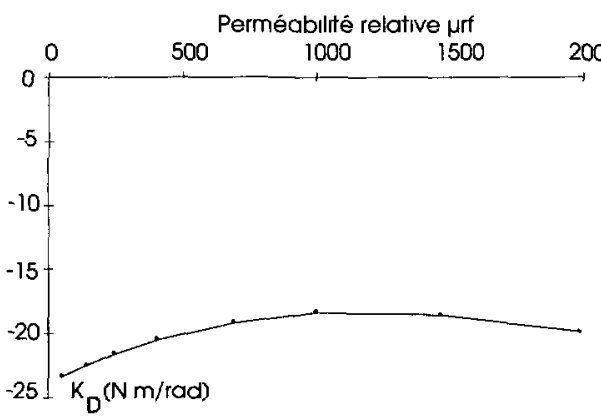

a)

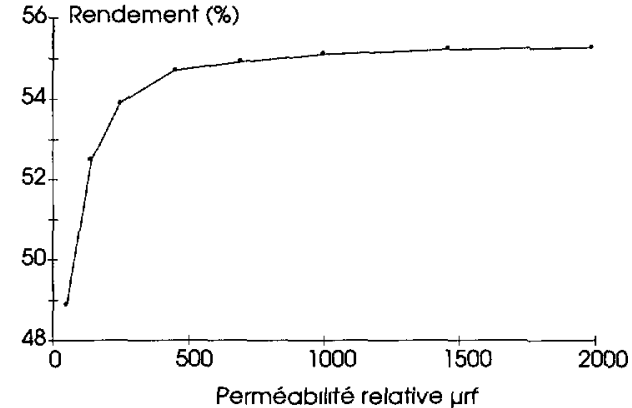

b)

Fig. 23 - Variation du facteur de démarrage limite (a) et du rendement électromagnétique (b) en fonction de la perméabilité relative du matériau magnétique.

[Varration of the limit starting factor (a) and of electromagnetic efficiency (b) as function of the magnetic material's relative permeability.]

comparativement à d'autres matériaux usuellement utilisés en électrotechnique tels que le fer silicium. De ce fait, nous avons étudié l'influence de $\mu_{\mathrm{rf}}$ sur les conditions de démarrage limites et sur les performances de la machine. Les courbes de la figure 23 montrent néanmoins qu'à partir d'une certaine valeur (environ 500), la valeur de $\mu_{\mathrm{rf}}$ est peu influente aussi bien sur les conditions de démarrage en monophasé que sur le rendement électromagnétique.

\section{Validation expérimentale}

Nous devons maintenant vérifier la validité des principales hypothèses et modélisations adoptées dans les précédents chapitres.

Nous présenterons la majorité des résultats sous forme d'histogrammes pour lesquels nous avons affecté, comme le présente le tableau suivant, un indice à chaque configuration de machines testées. Les caractéristiques principales des machines testées sont identiques aux caractéristiques de base données précédemment au tableau I.

4.1. RÉSISTANCE DE LA BOBINE . - La bobine que nous avons utilisée pour nos essais est commune aux différents prototypes. La mesure, réalısée en quatre fils, nous a donné à $20^{\circ} \mathrm{C}$ une valeur de $0,32 \Omega$.

Le coefficient de bobinage $K_{\mathrm{b}}$ est de 0,4 . La valeur de la résistance obtenue par calcul vaut $0,33 \Omega$, ce qui donne un écart faible de $3 \%$.

4.2. INDUCTANCE DE LA MACHINE ET PERMÉANCE VUE DU BOBINAGE. - Les valeurs théoriques et expérimentales sont pratiquement identiques, les écarts sont inférieurs à $5 \%$. Pour information, les résultats obtenus avec les machines 4 et 6 pôles, repère $\mathrm{m} 3$, sont présentés au tableau III.

4.3. AMPLITUde DE LA TENSION À VIDE. - Le relevé présenté à la figure 24, à la vitesse nominale de $15000 \mathrm{tr} / \mathrm{min}$ montre que la forme instantanée de celle-ci est quasi-sinusoidale. Entre les résultats théoriques et expérimentaux, présentés figure 25, les écarts, concernant la tension à vide crête à cette même vitesse n'excèdent pas $9 \%$. 
Tableau II. - Caractérıstıques dımensionnelles des prototypes testés.

[Dimensional characteristics of tested prototypes.]

\begin{tabular}{|c|c|c|c|c|c|}
\hline & \multicolumn{3}{|c|}{ MACHINE 4 PÔLES } & \multirow{2}{*}{\multicolumn{2}{|c|}{$\begin{array}{l}80^{\circ} \\
(s=0,44)\end{array}$}} \\
\hline $\begin{array}{l}\text { Largeur angulaire des dents } \\
\text { rotoriques }\end{array}$ & $\begin{array}{l}110^{\circ} \\
(s=0,61)\end{array}$ & $\begin{array}{l}90^{\circ} \\
(s=0,5)\end{array}$ & & & \\
\hline $\begin{array}{l}\text { Valeur de l'entrefer actif } E_{1} \\
\text { (avec } a=0,4 \text { ) }\end{array}$ & $\begin{array}{l}0,25 \mathrm{~mm} \\
(e=0,034)\end{array}$ & $\begin{array}{l}0,25 \mathrm{~mm} \\
(e=0,034)\end{array}$ & $\begin{array}{l}0,5 \mathrm{~mm} \\
(e=0.07)\end{array}$ & {$\left[\begin{array}{l}0,25 \mathrm{~mm} \\
(e=0,034)\end{array}\right]$} & $\begin{array}{l}0,5 \mathrm{~mm} \\
(e=0,07)\end{array}$ \\
\hline \multirow[t]{2}{*}{ Repère affecté à la machine } & m1 & $\mathbf{m} 2$ & $\mathrm{m3}$ & $\mathbf{m 4}$ & m5 \\
\hline & \multicolumn{3}{|c|}{ MACHINE 6 PÔLES } & & \\
\hline $\begin{array}{l}\text { Largeur angulaire des dents } \\
\text { rotoriques }\end{array}$ & $\begin{array}{l}80^{\circ} \\
(s=0,61)\end{array}$ & \multicolumn{2}{|l|}{$\begin{array}{l}60^{\circ} \\
(s=0,5)\end{array}$} & \multicolumn{2}{|l|}{$\begin{array}{l}53^{\circ} \\
(s=0,44)\end{array}$} \\
\hline $\begin{array}{l}\text { Valeur de l'entrefer actif } E_{1} \\
\text { (avec } a=0,4)\end{array}$ & $\begin{array}{l}0,25 \mathrm{~mm} \\
(e=0,034)\end{array}$ & $\begin{array}{l}0,25 \mathrm{~mm} \\
(e=0,034)\end{array}$ & $\begin{array}{l}0,5 \mathrm{~mm} \\
(e=0.07)\end{array}$ & $\begin{array}{l}0,25 \mathrm{~mm} \\
(e=0,034)\end{array}$ & $\begin{array}{l}0,5 \mathrm{~mm} \\
(e=0,07)\end{array}$ \\
\hline Repère affecté à la machine & $\mathrm{m} 1$ & $\mathbf{m 2}$ & $\mathrm{m3}$ & $\mathrm{m} 4$ & m5 \\
\hline
\end{tabular}

Tableau III. - Inductances mesurées et calculées.

[Measured and computed self inductance values.]

\begin{tabular}{|l|l|l|l|}
\hline Nombre de paire de pôles & Mesures & Calculs & Ecart \\
\hline$N_{\mathrm{p}}=2$ & $1,70 \mathrm{mH}$ & $1,63 \mathrm{mH}$ & $4,3 \%$ \\
\hline$N_{\mathrm{p}}=3$ & $1,95 \mathrm{mH}$ & $1,98 \mathrm{mH}$ & $1,5 \%$ \\
\hline
\end{tabular}

4.4. AMPlitude DU COUPLE DE DÉTENTE . - Les valeurs théoriques et expérimentales du couple de détente crête sont présentées figure 26. Les écarts maximaux sont inférieurs à $12 \%$. Cet écart est dû essentiellement au flux de fuites des aimants dans le plan axial de la machine non pris en compte lors de notre modélisation.

4.5. Amplitude du COUPle hyBRide. - Les histogrammes de la figure 27 font apparaitre les résultats expérimentaux et théoriques de la valeur crête du couple hybride, obtenue lorsque la force magnétomotrice de la bobine est égale à 400 puis $600 \mathrm{At}$. L'écart le plus important est inférieur à $15 \%$. Cet écart est dû en grande partie à un effet de saturation des pièces magnétiques et particulièrement de l'axe magnétique assurant le retour du flux.

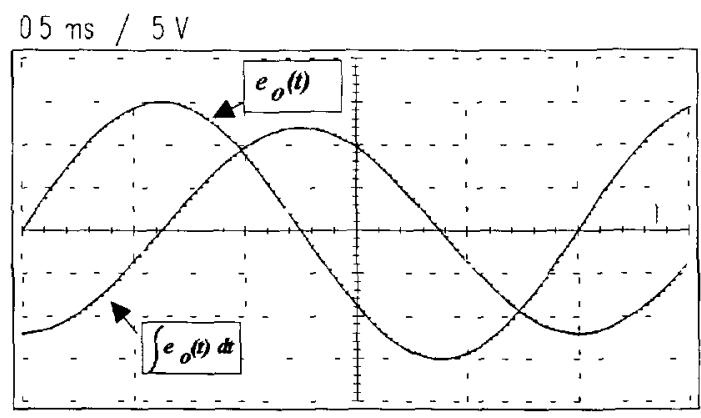

Fig 24. - Relevé de la f.e.m. à $15000 \mathrm{tr} / \mathrm{min}$.

[The measured e.m.f. at $15000 \mathrm{rpm}$.] 


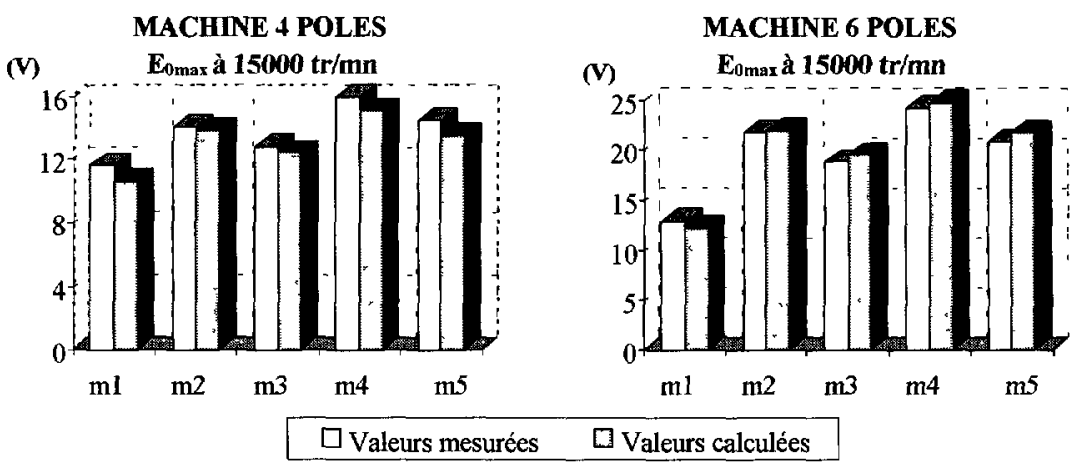

Fig 25. - Comparaison de l'amplitude de la tension à vide mesurée et de celle calculée. [Comparison of the measured and computed of e.m.f peak values.]

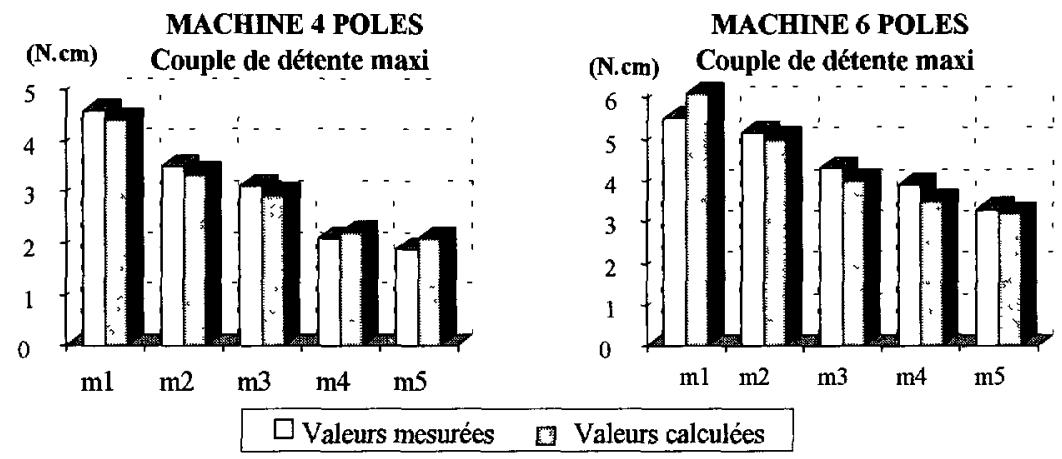

Fig 26. - Comparaison de l'amplitude du couple de détente mesuré et de celui calculé. [Comparison of the measured and computed of detent torque peak values.]

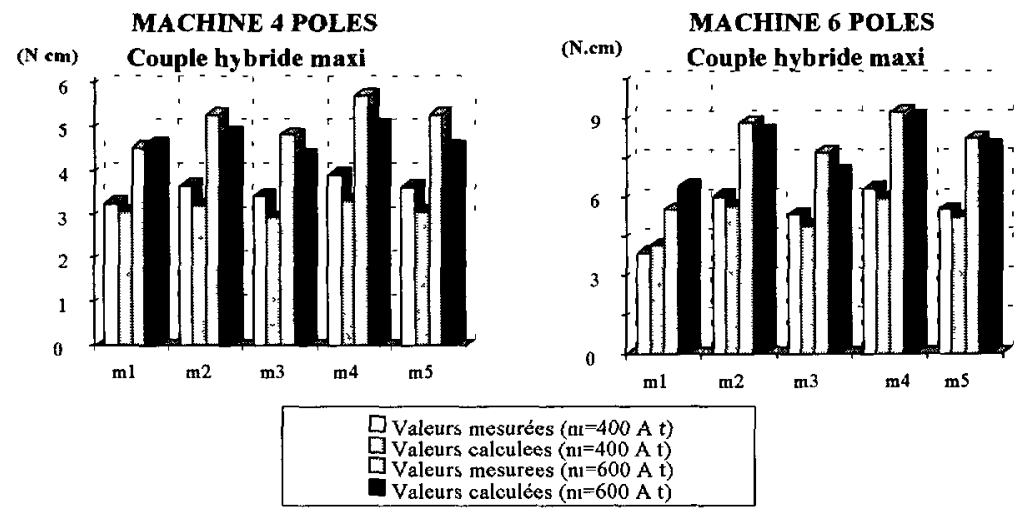

Fig. 27. - Comparasson de l'amplitude du couple hybride mesuré et de celui calculé pour nı $=400$ et $600 \mathrm{At}$.

[Comparison of the measured and computed of hybrid torque peak values for $\imath=6 \mathrm{~A}$ and $\imath=4 \mathrm{~A}$.] 


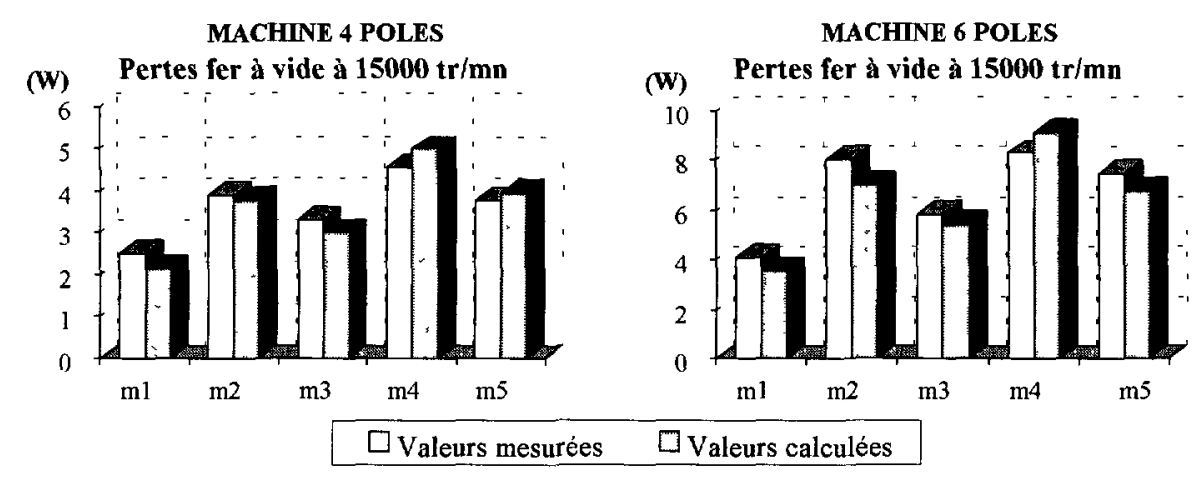

Fig. 28. - Comparaison des pertes fer à vide mesurées et de celle calculées à $15000 \mathrm{tr} / \mathrm{min}$ [Comparison of the measured and computed of no load uron losses at $15000 \mathrm{rpm}$.]

4.6. Pertes FER À vide . - Les valeurs des pertes fer à vide à $15000 \mathrm{tr} / \mathrm{min}$, relevées expérimentalement et obtenues par calculs, sont présentées figure 28 . L'écart maximum atteint est de $14 \%$. L'erreur obtenue est due d'une part, à la caractérisation du matériau magnétique. En effet, la formulation présentée dans la partie théorique de ce travail a été obtenue à partir de relevés effectués sur des échantillons de poudre de fer. Sachant que le circuit magnétique de la machine est obtenue par moulage à forte pression, les dispersions engendrées entre l'échantillon et le circuit fini sont par conséquent importantes. D'autre part, la formulation des pertes fer utilisée considère que la répartition de l'induction magnétique dans les différentes parties du circuit de retour est homogène.

\section{Conclusion}

Notre objectıf était de développer un modèle semi-analytique combinant précision, souplesse et rapidité d'exécution pour l'analyse paramétrique et le dimensionnement d'une machine monophasée à aimants alternés

La structure étudiée présente deux particularités. Tout d'abord, c'est une machine à bobinage global à rotor passif constitué de poudre de fer de réalisation très simple. D'autre part, le démarrage monophasé est obtenu sans dissymétrie magnétique.

Ces deux particularités imposent l'intégration dans les calculs de la structure magnétique complète et une détermination des couples de détente et hybride de manière précise. Ceci nécessite une modélisation prenant donc en compte l'aspect tridimensionnel et un calcul suffisamment précis des réluctances d'entrefer et inter-aimants.

Pour ces raisons, la zone active de la machine a été modélisée à partir d'une approche cellulaire permettant, sous certaines hypothèses, de définir un domaine normalisé où sont effectués les calculs par éléments finis. Ceci a permis de déterminer avec une bonne précision les valeurs des réluctances en fonction de la position du rotor. Notons également que la réluctance mutuelle entre aimants intégrée dans le schéma magnétique équivalent, sans laquelle les résultats obtenus sont peu satisfaisants, ne peut être calculée correctement qu'à l'aide d'une méthode numérique. Le circuit de retour du flux a, quant à lui, été pris en compte à l'arde d'une méthode analytique classique.

Les principales caractéristiques électromagnétiques de la machine ont ensuite été déterminées en fonction des différents paramètres géométriques et des caractéristiques des matériaux de la machine. 
Les résultats de cette étude ont montré notamment que la largeur angulaire des dents rotoriques permettant le démarrage en monophasé devra être supérieure à $70 \%$ de la largeur angulaire des aimants permanents $(s>0,35)$. Cette valeur limite est cependant dépendante de la hauteur relative des aimants $(a)$. L'utilisation d'aimants épais favorise sensiblement le démarrage mais aux dépens du rendement. Par ailleurs, la prise en compte des pertes Joule et fer dans le dimensionement de la machine a permis de montrer qu'il existe une valeur optimale de $a$ maximisant le rendement. La valeur normalisée obtenue dans le cas étudié est d'environ 0,35 .

D'autre part, les résultats de calcul concernant l'influence du jeu mécanique ont montré que l'augmentation de ce dernier est néfaste aussi bien pour le démarrage en monophasé que pour le rendement électromagnétique de la machine. Cependant, cette influence est intimement liée à la hauteur des aimants.

Enfin, l'étude effectuée a mis en évidence la faible influence de la perméabilité relative du matériau magnétique sur le démarrage et sur le rendement électromagnétique et ce à partir d'une certaine valeur relativement faible (égale à 500 dans le cas étudié). Notons que le matériau ferromagnétique isotrope utilisé a une perméabilité relative d'environ 140 .

La seconde partie du travail présenté a consisté à vérifier la validité du modèle et, par conséquent, des hypothèses mises en place. La comparaison des résultats expérimentaux avec ceux obtenus par calculs, pour les différentes configurations de prototypes, fait apparaître, selon les caractéristiques étudiées, un écart maximum compris entre 5 et $15 \%$.

Compte tenu des hypothèses simplificatrices mises en place aux différentes étapes de la modélisation (régime linéaire, effets d'extrémités négligés) et des incertitudes concernant la précision et la stabilité des caractéristiques des différents matériaux employés, ce résultat peut être considéré comme satisfaisant.

Cependant, une étude plus approfondie prenant en compte la non linéarité des caractéristiques magnétiques, des aimants non jointifs et la forme d'onde du courant réelle dans l'évaluation du facteur de démarrage reste à effectuer.

De plus, l'optimisation de l'induction rémanente des aimants permanents afin de maximiser le rendement de la machine compte tenue des caractéristiques de celle-ci est nécessaire. En effet, l'utilisation d'aimants à forte induction rémanente engendre l'accroissement excessif des pertes fer et donc un rendement électromagnétique faible. D'autre part, l'utilisation d'aimants à faible rémanence engendre, à même couple moteur, l'accroissement des ampères-tours (donc des pertes Joule) et par conséquent la diminution du rendement.

\section{Remerciements}

Nous remercions la société SAGEM S.A. pour sa collaboration dans la réalisation des prototypes et les moyens d'essai mis à notre disposition, ainsi que l'équipe "matériaux magnétiques" du LESIR pour la caractérisation de la poudre de fer.

\section{Annexe}

Expression des termes de perméance $a_{1}, a_{2}, b, m_{\mathrm{a}_{1} \mathrm{~b}}, m_{\mathrm{a}_{2} \mathrm{~b}}$ et $m_{\mathrm{a}_{1} \mathrm{a}_{2}}$ :

$$
\begin{array}{ll}
D a_{1}=\left[\left(R_{\mathrm{c} 2}+R_{\mathrm{c} 3}\right) K_{1}+K_{2}\right] & D a_{2}=\left(R_{\mathrm{c} 1}+R_{\mathrm{c} 3}\right) K_{1}+K_{2} \\
D m_{\mathrm{a}_{1} \mathrm{~b}}=\left(K_{3} R_{c 2}\right) & D m_{\mathrm{a}_{2} \mathrm{~b}}=-\left(K_{3} R_{\mathrm{c} 1}\right) \\
D m_{\mathrm{a}_{1} \mathrm{a}_{2}}=\left(K_{1} R_{\mathrm{c} 3}+K_{2}\right) K_{1}+K_{2} & D b=\left(R_{\mathrm{c} 1}+R_{\mathrm{c} 2}\right)\left(\frac{R_{\mathrm{c} 3}}{4}+K_{4}\right)+\left(\frac{R_{\mathrm{c} 1} R_{\mathrm{c} 2}}{4}\right)
\end{array}
$$

avec :

$$
D=K_{1}\left(R_{\mathrm{c} 1} R_{\mathrm{c} 2}+R_{\mathrm{c} 1} R_{\mathrm{c} 3}+R_{\mathrm{c} 2} R_{\mathrm{c} 3}\right)+K_{2}\left(R_{\mathrm{c} 1}+R_{\mathrm{c} 2}\right)
$$




$$
\begin{aligned}
K_{1} & =R_{2}+R_{\mathrm{fb}} \\
K_{2} & =R_{1} R_{2}+R_{2} R_{3}+R_{\mathrm{fb}}\left(R_{1}+R_{2}+R_{3}\right) \\
K_{3} & =\frac{1}{2}\left(R_{2}+2 R_{\mathrm{fb}}\right) \\
K_{4} & =\frac{1}{4}\left(R_{1}+R_{2}+R_{3}+4 R_{\mathrm{fb}}\right)
\end{aligned}
$$

\section{Bibliographie}

[1] Jufer M., Électromécanique (Édition DUNOD, 1979).

[2] Lacroux G., Les aimants permanents (Éditions Technique et Documentation, Lavoisier 1989).

[3] Prevond L., Étude, modélisation et expérimentation de structures hybrides inovantes tournantes et linéarres, Thèse de doctorat CNAM Paris, décembre 1994.

[4] Lucidarme J., Multon B. et Prevond L., Actionneurs hybrides monophasés à commutation de flux, Brevet Français CNRS, $n^{\circ}$ F 94/12063, octobre 1994. Demande de Brevet Européen n ${ }^{\circ}$ 93402266.1, octobre 1995.

[5] Ben Ahmed A., Contribution à la conception des machines synchrones à aimants permanents et à bobinage global, Thèse de doctorat Paris VI, janvier 1994.

[6] Ben Ahmed A., Lucidarme J. et Desesquelles P.F., Méthode semi-numérique de pré-dimensionnement des machines à aimants permanents et à bobinage global, J. Phys. III France 5 (1995) 703-725.

[7] Desesequelles P.F., Lucidarme J. et Ben Ahmed A., Theoretical and Experimental Results Upon Multi-airgap Axial Synchronous Machines With Permanent Magnets, I.C.E.M 90, Cambridge Ma. (USA). August 1990.

[8] Aufauvre E., Étude et réaliation d'un moteur hybride monophasé grande vitesse., Mémoire Ingénieur CNAM, mars 1996.

[9] Benghezal K., Ivanes M. et Yonnet J.P., Étude d'un moteur synchrone à almants permanents par simulation numérique, Journée SEE : Les actionneurs électriques, esclaves des temps modernes, organisées par SEE, Valence 1989.

[10] Muller R., Collector-less DC motor, US Patent 3 873 897, 1975.

[11] Altenberg G., Actual aspects of the development of fractional horse-power single phase synchronous motors with permanent magnet, SM'100, Part III, Zurich, August 1991, pp. 1083-1089.

[12] Henry-Baudot J. et Lacroux G., Petits moteurs électriques, Technique de l'ingénieur, traité de génie électrique, D545, 1980.

[13] Kant M., Les actionneurs électriques pas à pas (Édition Hermès, 1989).

[14] Grellet G., Pertes dans les machines tournantes, Techniques de l'Ingénieur, D3450.

[15] Geoffroy M., Multon B. et Hoang E., Couplage de méthodes pour le calcul rapide des caractéristiques électromagnétiques des machines à réluctance variable à double saillance, Colloque méthodes informatiques de la conception industrielle, ESIM Marseille, juin 1993, pp. 81-90.

[16] Rossi R.J.C., Machine électrique à entrefers radiaux, Brevet Européen $\mathbf{n}^{\circ}$ EP 312-464-A1, 1988.

[17] Roisse H. et Brochet P., Dynamic simulation of a synchronous permanent magnet machine using the permeance network method, Electrimacs'96, Saint-Nazaire, septembre 1996, pp. 1107-1112.

[18] Delforge C. et Lemarre-Semail B., Induction machine modelling using finite element method and permeance network method, IEEE Trans. on Magnetıcs 31 (1995) 2092-2095. 